\title{
Gut microbiota changes in the extreme decades of human life: a focus on centenarians
}

\author{
Aurelia Santoro ${ }^{1,2} \cdot$ Rita Ostan $^{1,2} \cdot$ Marco Candela $^{3} \cdot$ Elena Biagi $^{3} \cdot$ Patrizia Brigidi $^{3}$. \\ Miriam Capri $^{1,2} \cdot$ Claudio Franceschi $^{4}$
}

Received: 25 September 2017 / Accepted: 29 September 2017 / Published online: 14 October 2017

(c) The Author(s) 2017. This article is an open access publication

\begin{abstract}
The gut microbiota (GM) is a complex, evolutionarily molded ecological system, which contributes to a variety of physiological functions. The GM is highly dynamic, being sensitive to environmental stimuli, and its composition changes over the host's entire lifespan. However, the basic question of how much these changes may be ascribed to variables such as population, diet, genetics and gender, and/or to the aging process per se is still largely unanswered. We argue that comparison among studies on centenarians - the best model of healthy aging and longevity—recruited from different geographical areas/populations (different genetics and dietary habits) can help to disentangle the contribution of aging and non-aging-related variables to GM remodeling with age. The current review focuses on the role of population, gender and host genetics as possible drivers of GM modification along the human aging process. The feedback impact of age-associated GM variation on the GMbrain axis and GM metabolomics is also discussed. We likewise address the role of GM in neurodegenerative diseases
\end{abstract}

Aurelia Santoro and Rita Ostan contributed equally to this work.

Aurelia Santoro

aurelia.santoro@unibo.it

1 Department of Experimental, Diagnostic and Specialty Medicine (DIMES), Alma Mater Studiorum-University of Bologna, Via San Giacomo 12, 40126 Bologna, Italy

2 Interdepartmental Centre "L. Galvani” (CIG) Alma Mater Studiorum-University of Bologna, Via San Giacomo 12, 40126 Bologna, Italy

3 Department of Pharmacy and Biotechnology (FABIT), Alma Mater Studiorum-University of Bologna, Via Belmeloro 6, 40126 Bologna, Italy

4 Institute of Neurological Sciences (IRCCS), Via Altura 3, 40139 Bologna, Italy such as Parkinson's and Alzheimer's, and its possible therapeutic use, taking advantage of the fact that centenarians are characterized by an extreme (healthy) phenotype versus patients suffering from age-related pathologies. Finally, it is argued that longitudinal studies combining metagenomics sequencing and in-depth phylogenetic analysis with a comprehensive phenotypic characterization of centenarians and patients using up-to-date omics (metabolomics, transcriptomics and meta-transcriptomics) are urgently needed.

Keywords Gut microbiota $\cdot$ Aging $\cdot$ Centenarians $\cdot$ Gutbrain axis $\cdot$ Host genome

\section{The study of the human gut microbiota: methodological aspects}

The human gut microbiota (GM) is a highly diverse ecosystem made up of trillions of bacteria populating the gastrointestinal tract. This niche establishes a complex, multispecies apparatus in which every occupant plays a role and modulates its own activity in response to signals coming from inside and outside the human host [1]. The composition of the GM is affected by a plethora of individual, population and environmental variables, e.g., age, gender, genetic background, biography (type of delivery, breastfeeding or formula feeding, use of antibiotics), immuno-biography (lifelong immunological experience) and geography (ethnicity, cultural habits, nutrition). These factors over a lifetime impinge on the GM, resulting in huge variability and heterogeneity of this ecosystem in human beings. This adaptive nature of the GM is functional to calibrating the immune and metabolic pathways in response to individual needs, and has a profound impact on health and disease. Indeed, the GM has emerged as a dynamic community able to adapt its 
composition and functionality to the varying conditions in which the human host lives to meet the changing demands of host metabolism [2]. Thus, a healthy adult GM structure is properly defined as a set of many possible configurations which, even when differing in composition, share a comparable degree of diversity and evenness (meaning the number of species with an equal distribution in the ecosystem), and the ability to preserve the homeostasis of the human host [3] . In this elaborate scenario, the most informative approach for understanding the role of the GM in its lifelong maintenance of host homeostasis would clearly be by longitudinal studies monitoring individuals over time (years and decades) to identify and follow the specific trajectories of their agerelated GM modifications. To date, this kind of analyses has not been possible because attention towards the GM is quite a recent development, while the most reliable and robust longitudinal studies have not collected stool samples across the full life span of individuals. Hopefully, new life-long longitudinal studies or continuations of existing ones will cover this gap.

At present, the best way of grasping the adaptive pattern of human GM as humans age is represented by crosssectional studies embracing a wide age range in well-defined populations that are relatively homogeneous in genetics and lifestyles. Inclusion of "extreme phenotypes", i.e., individuals who are at the extreme ends of a trait distribution (healthy subjects versus patients suffering from diseases), can help in identifying specific signatures within overall age-related trajectories, regarding genetics, epigenetics, metabolomics, and including metagenomics, among other things [4-8]. Such is the case of centenarians who represent a clearly defined and highly informative "super-control" group, since, unlike younger controls, most of them achieved their remarkable age by avoiding or perhaps postponing major age-related diseases. The strategy of focusing on individuals from welldefined populations and including the "extreme phenotypes" such as centenarians increases one's power to identify physiological age trajectories, including the last 20-30 years of human life which are usually neglected [9]. Comparison between data sets obtained from different populations will allow us to disentangle changes related to specific genetic or lifestyle habits, including diet, from changes related to the aging process itself.

\section{The model of centenarians}

Centenarians represent the best model of "successful" aging showing a lower incidence of chronic illness, a reduction of morbidity and an extension of health span in comparison to octogenarians and nonagenarians from the same cohort [10, 11]. Thus, the study of the GM of exceptionally long-lived individuals is providing insights into how the GM successfully adapts in an extremely long lifespan to the progressive age-related environmental (lifestyle, diet, etc.) and endogenous changes, contributing to the maintenance of metabolic and immunological homeostasis and promoting survival [1, 8].

Human longevity has a strong familial and genetic component $[12,13]$. Data from different populations have shown that relatives (parents, siblings and offspring) of long-lived subjects have a significant survival advantage, a higher probability of being or becoming long-lived and a lower risk of undergoing major age-related diseases [14-17]. Family genealogy data from Sardinian centenarian women have confirmed that maternal longevity is associated with lower infant mortality in offspring [18] suggesting that parents/ mothers who will later become centenarians very likely adopt healthier lifestyles for their children. Considering that the study of centenarians has some obvious limitations (rarity, lack of an age-matched control group and frailty related to extreme age), centenarians' offspring, representative of the elderly age bracket whose lifestyle can still be modified to attain better health, may provide a useful model to study both genetic and environmental/lifestyle determinants of healthy aging [14].

Starting from observation of the profound changes in immune responses with age (immunosenescence, i.e., the overall age-related remodeling of the immune system [19]) and taking into account the increasing amount of experimental data on genetics, proteomics, epigenetics, metabolomics, glycomics, etc. [20], one may conceptualize the aging process as a continuous lifelong remodeling of the whole human organism [21]. The exceptional phenotype of centenarians has been revealed as unexpectedly complex and very dynamic, being a unique mixture of adaptive robustness and accumulating frailty [21-26] resulting from the ability of the centenarian's organism to respond/adapt to damaging stimuli.

According to the dynamics of world population aging, a lifelong approach including the last decades of life is extremely important if we are to understand the basis of the longevity process, considering that the oldest-old are the fastest growing segment of the population in many countries. It is also interesting to note that the birth cohort is crucial in the health outcome of long-lived people. A comparison of two Danish cohorts born 10 years apart (1905 and 1915) showed that the younger cohort had longer survival and scored significantly better on both cognitive tests and the activities of daily living scale than the cohort born in 1905, despite being 2 years older at the time of assessment. This finding suggests that more people are living to older ages with better overall functioning [27].

Demographic projections suggest that there will be 3.7 million centenarians across the globe in 2050. In particular, China is expected to have the largest centenarian population, followed by Japan, the United States, Italy and India. In this 
scenario, the global number of persons aged 80 or over is projected to increase from 125 million in 2015 to 434 million in 2050 with a dramatic hike of the resources needed to care for them [28].

\section{Gut microbiota from birth to 100 years and beyond}

The programming of immune response and metabolic pathways is heavily influenced by the interaction between the human organism and its GM starting from infancy. This bidirectional relationship in early life has a profound impact on health and disease in later life.

A very recent paper has proposed that the progressive process of microbial colonization of the human ecosystems may be initiated in utero by the microbial populations of the maternal placenta and amniotic fluid which share some features with the microbiota detected in infant meconium [29]. Moreover, during vaginal delivery, a considerable inoculum of maternal intrauterine microbes is received by the neonate and, after birth, neonatal gut colonization is continued by microbes present in maternal milk and feces, with human milk factors (e.g., complex polysaccharides and antibodies) selectively promoting the growth of mutualistic microbial partners. Thus, antibiotic exposure during pregnancy, cesarean section delivery, postnatal antibiotic administration, and formula feeding may all alter the early intestinal microecology, and these factors have been associated with the risk of disease in later life [30-34]. These findings reveal that the aging process could also depend on early stimuli and experiences that may exert long-term effects. To the best of our knowledge, no studies have correlated the physiological and/ or pathological phenotype of elderly and extremely old individuals with these initial events shaping the early GM establishment. For instance, in centenarian databases no data are available on the mode of delivery, breast- or other types of feeding (wet nurse, animal milk, etc.), nutrition and hygienic conditions in the early years of life. Historical anthropology studies could shed light on these points.

Starting from life in utero, the gastrointestinal tract is colonized by a wide range of bacteria of maternal, dietary and environmental origin, which, after assembling themselves into a highly interconnected bacterial community, co-operate in several vital host functions, including nutrient digestion and absorption, immune function, as well as the development of an appropriate stress response. This close symbiotic relationship makes humans inter-dependent "meta-organisms" [35, 36], where the commensal bacteria function as a metabolic and endocrine organ [37] and, in turn, the human immune system has properly evolved to control the physiological life-long low-grade inflammatory response triggered by the GM.
The human GM metacommunity is estimated to consist of over 1000 different microbial species [38] belonging to 5 predominant phyla: Firmicutes and Bacteroidetes followed by Actinobacteria, Verrucomicrobia and Proteobacteria [39, 40]. As previously discussed, the GM is a malleable ecosystem, being able to adapt its phylogenetic and functional profile to changes in diet, environment, lifestyle, antibiotic treatments and stress. In a mutualistic context, this plasticity is functional to optimizing the metabolic and immune performance of the host in response to environmental and physiological changes, preserving physiological homeostasis and health status [41].

The human GM is a complex and dynamic environment, which undergoes profound life-long remodeling, sometimes with a concrete risk of maladaptive changes. Indeed, in certain circumstances the age-related pathophysiological changes in the gastrointestinal tract, modification of lifestyle, nutrition [42] and behavior, as well as immunosenescence and "inflammaging" (the chronic low-grade inflammatory status typical of the elderly, [23]) strongly impact on the GM, eventually forcing maladaptive variations [43]. Inflammation, in particular, may result in a higher level of aerobiosis and production of reactive oxygen species that inactivate the strict anaerobic Firmicutes, while allowing a bloom of facultative aerobes, as frequently observed in the elderly [41]. These microorganisms (i.e., Enterobacteriaceae, Enterococcaceae, Staphylococcaceae), generally called "pathobionts", can prosper in an inflamed gut as they are relatively oxygen tolerant, getting the better of mutualistic symbionts and further supporting inflammation [44]. On the other hand, these age-related GM changes can compromise the host immune homeostasis in favor of a proinflammatory profile creating a vicious inflammatory circle and may contribute to the progression of diseases and frailty in the elderly [45-47]. Frailty has been negatively associated with GM diversity [48] and Eubacterium dolichum and Eggerthella lenta have been found to be more abundant among frail individuals, while Faecalibacterium prausnitzii was less abundant, thus identifying a GM signature of frailty [48]. A very recent publication has demonstrated that germfree mice are protected from inflammaging [49]. When these mice are co-housed with old, but not young, mice the levels of pro-inflammatory cytokines in the blood increase together with intestinal permeability and macrophage dysfunction [49]. On the whole, these data prove that age-related dysbiosis is responsible for the age-related increase in systemic inflammation. Thus, pursuing a wholesome and adaptive GM trajectory during aging is dramatically emerging as a key factor in the achievement of healthy aging and maintenance of host homeostasis [50].

The comparison of GM among young adults, the elderly, and centenarians has highlighted that the mutualistic changes in the composition and diversity of the gut ecosystem do not 
follow a linear relation with age, remaining highly similar between young adults and 70-year-olds and markedly changing in centenarians. Thus, the GM seems to rest in a stable state from the third to the seventh decade of life [51], while after 100 years of symbiotic association with the human host, it shows a profound, and possibly adaptive, remodeling. Further analyses are needed to fill in the age gap between 70 and 100 years of age and complete the re-construction of age-related GM modifications.

Centenarians stand out as a separate population, their GM showing high diversity in terms of species composition (Table 1) [52]. Bacteroidetes and Firmicutes still dominate the GM of centenarians, but Firmicutes subgroups go through specific changes with a decrease in the contributing Clostridium cluster XIVa, an increase in Bacillus species, and a rearrangement of the Clostridium cluster IV composition. Several butyrate producers (Ruminococcus obeum et rel., Roseburia intestinalis et rel., Eubacterium ventriosum et rel., Eubacterium rectale et rel., Eubacterium hallii et rel., Papillibacter cinnamovorans et rel., and Faecalibacterium prausnitzii et rel.) were found in lower amounts, while others (Anaerotruncus colihominis et rel. and Eubacterium limosum et rel.) increased in centenarians, suggesting the existence of bacteria characteristic of longevity [51].

The GM of centenarians is enriched in facultative anaerobe bacteria mostly belonging to Proteobacteria which have been redefined as "pathobionts" because, in some circumstances, e.g., inflammation, they may escape surveillance, prevail over mutualistic symbionts and induce pathology $[44,53]$. The age-related remodeling of GM (i.e., proliferation of opportunistic Proteobacteria at the cost of symbiont Firmicutes and Bacteroidetes) may contribute to inflammaging and/or is affected by the systemic inflammatory status in a sort of self-sustaining loop. Indeed, the changes in GM profile observed in centenarians correlate with an increase in pro-inflammatory cytokines in the peripheral blood. In particular, these exceptionally long-lived subjects show high levels of IL-6 and IL-8, which correlate with an enrichment in Proteobacteria and a decrease in the amount of certain butyrate-producing bacteria [51].

A recent paper reconstructs the longest human microbiota trajectory with age by phylogenetic GM analysis of a sizable number of Italian young, elderly and extremely long-lived subjects (centenarians and semi-supercentenarians, i.e., persons who reach the age of 105 years) [8]. According to the authors, a core GM comprised of dominant symbiotic bacterial taxa (Ruminococcaceae, Lachnospiraceae, Bacteroidaceae) loses diversity and relative abundance of its members with age, thus decreasing in size. In extreme longevity, this shrinkage is counterbalanced by an increase in longevity-adapted and possibly health-promoting subdominant species (e.g., Akkermansia, Bifidobacterium, Christensenellaceae) as well as in their co-occurrence network. In addition, the GM of semi-supercentenarians is invaded by micro-organisms typical of other niches, such as Mogibacteriaceae and Synergistaceae, known to be abundant in the periodontal environment. In extremely aged people, centenarians and semi-supercentenarians, an overall increase has been observed in the GM diversity. Thus, while extremely aged people lose some of the most important core components of the adult GM, they acquire in parallel a wealth of new microbial GM components, including potential pathobionts and allochthonous microorganisms. Along with extreme aging, it seems that the host tolerates the consolidation of new GM ecosystem balances in the gut, resembling a property typical of the ancestral human GM $[54,55]$. In particular, to understand the GM-host's co-evolutionary trajectory, several studies have been conducted comparing the GM ecosystem of small-scale rural societies and that found in a westernized lifestyle [54]. This comparison revealed specific GM adaptations to the respective subsistence strategies, including higher diversity and enrichment in microorganisms generally considered as pathobionts (e.g., Prevotella, Treponema, Bacteroidetes and Clostridiales) in the GM from ancestral populations [56, 57]. For instance, the GM of Hadza hunter-gatherers from Tanzania showed a unique enrichment in metabolic pathways that align with dietary and environmental factors peculiar to their foraging lifestyle, characterized by a broad-spectrum carbohydrate metabolism, reflecting the complex polysaccharides in their diet during the rainy season, though it is also equipped for the branched-chain amino acid degradation and aromatic amino acid biosynthesis typical of their diet during the dry season $[55,58]$. Such research makes us appreciate the coadaptive functional role of the GM in complementing human physiology.
Table 1 Changes to the index of GM diversity in centenarians according to different papers

\begin{tabular}{|c|c|c|c|c|c|}
\hline \multirow[t]{2}{*}{ Diversity } & \multicolumn{5}{|c|}{ Change in centenarians according to } \\
\hline & Biagi et al. [51] & $\begin{array}{l}\text { Biagi } \\
\text { et al. } \\
{[1]}\end{array}$ & $\begin{array}{l}\text { Wang } \\
\text { et al. } \\
{[61]}\end{array}$ & Kong et al. [52] & $\begin{array}{l}\text { Odamaki } \\
\text { et al. [70] }\end{array}$ \\
\hline Simpson Reciprocal Index of Diversity & $\downarrow$ & $\uparrow$ & & & \\
\hline Alpha diversity (Chao Index) & & $\uparrow$ & $\uparrow$ & $\uparrow$ & $\uparrow$ \\
\hline Shannon Index & & $\uparrow$ & $=$ & $\uparrow$ & $\uparrow$ \\
\hline
\end{tabular}


Along with these studies, we can thus hypothesize that extremely long-lived people are able to rearrange the "mutualistic pact" with the GM, at least partly changing the microbial partners which support host health and physiology. It remains to be seen how these persons achieve this goal, and if and which environmental and/or genetic host factors are involved in this highly adaptive human process.

\section{Gut microbiota in centenarians from different continents: Italians versus Chinese and Japanese}

In studying the age-related remodeling of the human GM, one of the most challenging aspects is to discriminate effects due to the aging process per se from those due to the modification of diet and lifestyle that aging entails [3]. In advanced age, tooth loss, chewing and swallowing problems, impaired sense of taste and smell and reduced physical activity strongly affect the quality of diet and lifestyle $[59,60]$ and these, in turn, are very well known short- and long-term determinants impacting on GM composition and functionality [3].

One effective strategy to disentangle these aspects is to compare elderly and long-lived people with different nutritional habits, lifestyles and cultures. Thus, comparison between GM of Italian centenarians/semi-supercentenarians and Chinese old people (including centenarians) led to identification of gut-microbial signatures during healthy aging [52]. The combination of the two datasets suggests significant differences in community membership and structures between the Italian and Chinese long-living groups that can be attributed to geographic, genetic and nutritional factors (Table 2). However, common features such as to discriminate long-lived from young people were identified in both groups [52]. Finally, the GM of the long-living groups in both the Italian and Chinese cohorts is also enriched in Ruminococcaceae, Akkermansia and Christensenellaceae which have been classified as potentially beneficial bacteria and linked to body mass index, immunomodulation and healthy homeostasis [52].

Another paper, presenting the Illumina sequencing of 16S rRNA gene amplicons performed on the GM of centenarians living in one of the most long-lived villages in the world (Bama County, China), confirmed that the GM of centenarians was more diverse (count of the unique OTU numbers, Chao 1 index) than that of the younger elderly [61] (Table 1). The diversity of the GM community is considered as a key health indicator since it markedly affects the health status of the hosts, while a reduced GM diversity has been associated with several pathological conditions, including autoimmunity (inflammatory bowel disease and psoriatic arthritis), antibiotic treatment, Clostridium difficile infections, obesity and other metabolic alterations [62-69]. These results contrast with previous studies suggesting that the microbial diversity of GM was significantly reduced in centenarians [51]. However, in Biagi et al. [51] the GM was characterized by a microarray-based approach, making it impossible to fully characterize any unexpected diversity of the human GM ecosystem. Among the distinctive features of the fecal microbial communities of Bama County centenarians, the authors showed certain similarities (abundance of Escherichia, reduction in Bacteroidetes, structural change in butyrate-producing bacteria in the Clostridium cluster IV and Clostridium cluster XIVa) and some differences (low level of Akkermansia) with Italian centenarians (Table 2).

The paper by Odamaki et al. [70] provides a picture of the changes in the GM composition throughout human life, from birth to extreme aging in a large cohort of Japanese individuals [70]. However, even though children, adults and the elderly were abundantly represented, this analysis was not centered on longevity, including only six centenarians (100-104 years old) and seven over-95 year-olds. Importantly, a decrease in Faecalibacterium, Roseburia, Coprococcus, Blautia and an increase in Enterobacteriaceae were shown in 90- and 100-year-old subjects, resembling the agerelated microbiota features found in Italian centenarians but with some differences from Chinese centenarians (Table 2). Regarding the microbiota diversity, in the Japanese cohort the alpha diversity score and the Shannon index remained stable during adulthood and then increased in the elderly and centenarians, the later data confirming previous observations (Table 1) [70].

GM remodeling with age matches metabolome variations. Thus, centenarians showed a distinct metabolic pattern. A unique alteration of specific glycerophospholipids and sphingolipids [71] and decreased circulating levels of 9-hydroxy-octadecadienoic acid (9-HODE) and 9-oxo-octadecadienoic acid (9-oxoODE), markers of lipid peroxidation [7], are seen in the longevity phenotype in Italy. It has also been revealed that the longevity process deeply affects the structure and composition of the human GM-derived metabolome, as shown by the increased excretion of phenylacetylglutamine (PAG) and p-cresol sulfate (PCS) in Italian centenarians' urine [7]. In 647 individuals from the US, followed up for as much as 20 years, higher concentrations of the citric acid cycle intermediate, isocitrate, and the bile acid, taurocholate, were associated with lower odds of longevity, defined as attaining 80 years of age. In a larger cohort of 2327 individuals with metabolite data available, higher concentrations of isocitrate but not taurocholate were also associated with worse health conditions [72]. On the other hand, centenarians from the Bama County in China showed decreased levels of PCS but increased levels of fecal shortchain fatty acids (SCFAs) and total bile acids [73]. Intestinal commensal bacteria metabolize host-derived bile salts [74]. Bile acids are hormones that regulate their own synthesis, transport, glucose and lipid homeostasis and energy balance 


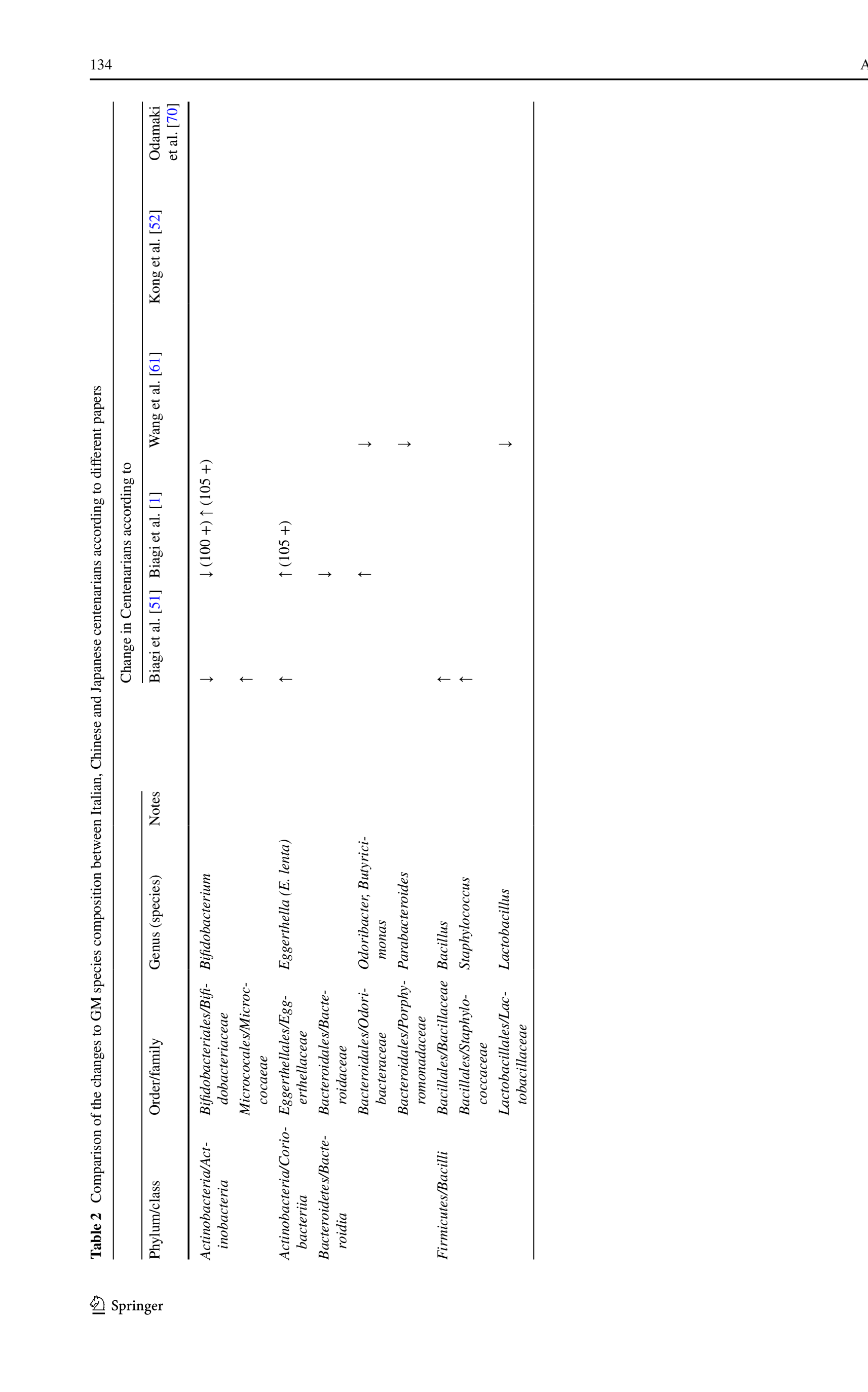




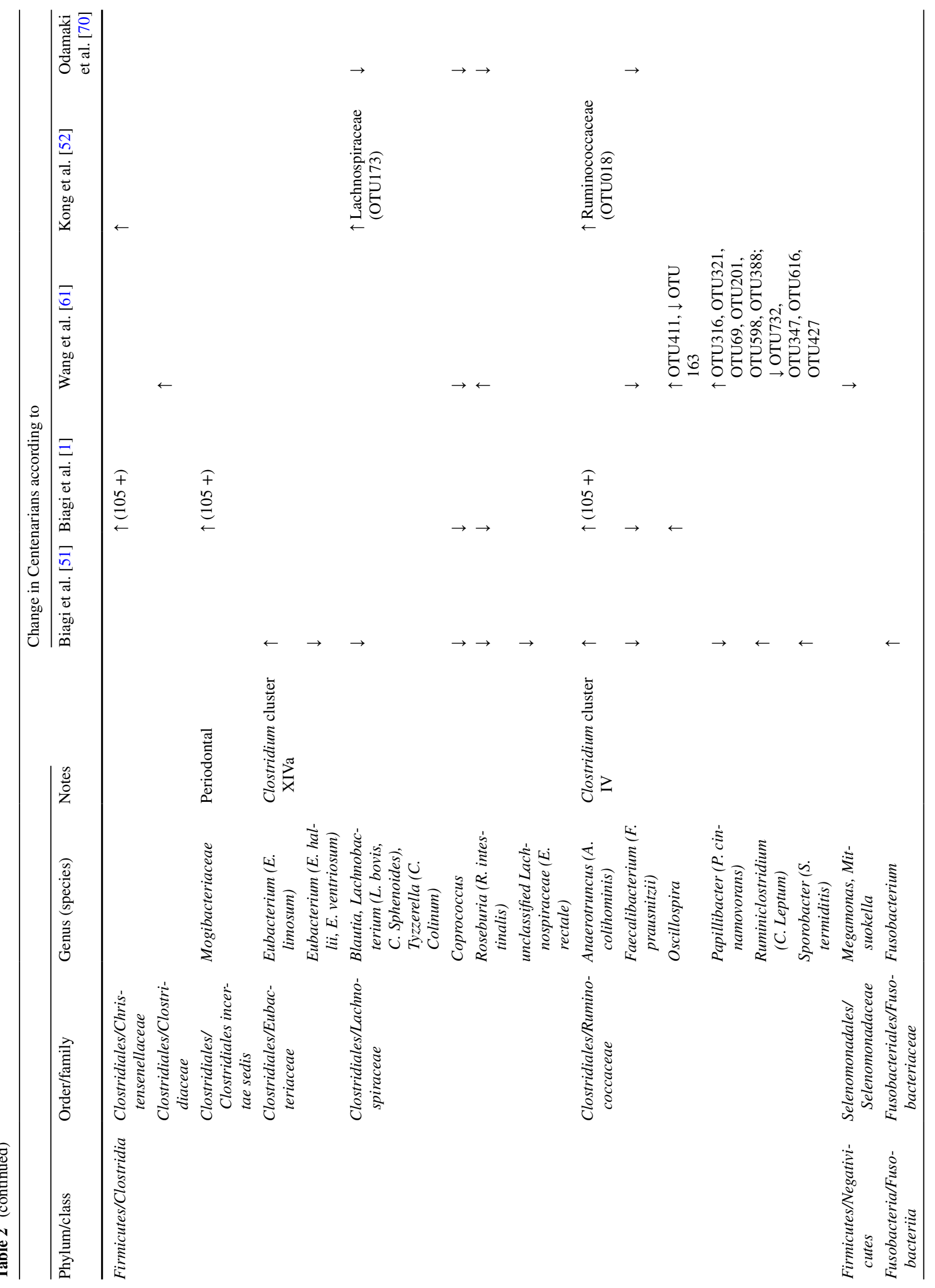




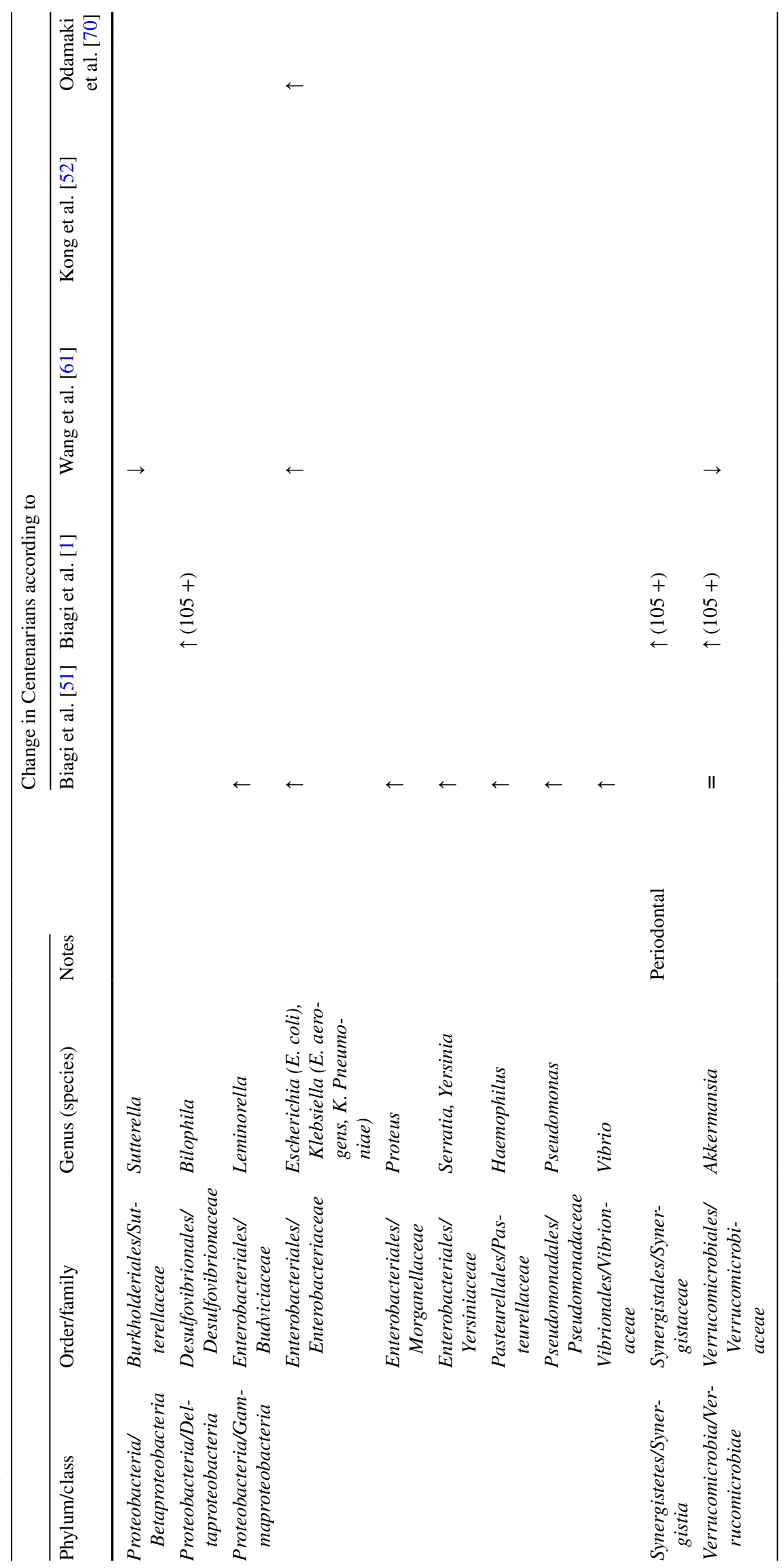


via activation of specific nuclear receptors and $\mathrm{G}$ proteincoupled receptors. The circulating bile acid pool composition consists of primary bile acids produced from cholesterol in the liver, and secondary bile acids formed by specific gut bacteria. The gut microbial community, through its capacity to produce bile acid metabolites distinct from the liver (i.e., secondary bile acids), can be thought of as an "endocrine organ" with the potential to alter host physiology, perhaps in their own favor. The term "sterolbiome" [74] describes the genetic potential of the GM to produce endocrine molecules from endogenous and exogenous steroids in the mammalian gut. Thus, changes in age-associated microbiome composition could impact on bacterial metabolism of steroid compounds and ultimately steroid hormones in peripheral tissues. Chinese centenarians have high levels of bile acids [73], suggesting a pro-longevity role. However, studies on different populations reported that increased levels of secondary bile acids are associated with an increased risk of age-associated diseases [72] and specific diseases of the gastrointestinal tract system [75].

On the whole, these data indicate that human GM alterations during aging are not univocal (Table 2) but follow different trajectories depending on lifestyle, nutrition, geographic/population/social factors as well as host genetics. In extremely long-lived people the composition, functionality and diversity of this complex and dynamic microbial community seem to achieve a peculiar balance resulting from a continuous 100-year remodeling process. Thus, it still remains to be determined how and if this (optimally?) adapted GM contributes to the homeostasis of the aged host, enabling him/her to reach the extreme limits of human life.

\section{Gut microbiota age-related changes, brain functions and neurodegenerative diseases}

To this already complex scenario, it should be added that the gastrointestinal tract establishes a strong bidirectional connection with the Central Nervous System (CNS) named the "gut-brain axis", along which the GM plays a crucial role. A number of experimental observations have shown that even mild alterations in GM composition are able to cause modification of cerebral functions, while conversely the brain can deeply affect intestinal functions via the secretion of hormones, neuropeptides and neurotransmitters such as substance $\mathrm{P}$, neurotensin, corticotropin releasing hormone, 5-hydroxytryptamine, and acetylcholine. The literature on this hot topic is extensive and more details can be found in recent reviews [76, 77]. Here, specific topics relating to the impact of GM age-related changes on brain physio-pathology, with particular attention to the role of tryptophan, will be briefly addressed. Gut and the GM affect brain and upper cognitive functions by two distinct pathways: (1) a direct one via retrograde stimulation of the Vagus nerve and the production of hormones and cytokines such as IL- 6 , TNF- $\alpha$ and VIP; (2) an indirect one, via the production of bacterial components and metabolites. The main microbial bioactive molecules are: proteins that may cross-react with human antigens and stimulate abnormal responses by the immune system [78, 79]; neurotoxic metabolites such as D-lactic acid and ammonia which are able to cross the blood-brain barrier and cause neurotoxicity or neuroinflammation [80-82]; hormones and neurotransmitters interfering with those of human origin (e.g., Lactobacillus and Bifidobacterium species are GABA neurotransmitter producers, Escherichia, Streptococcus and Enterococcus are serotonin synthesizers) [83-85]. Hence, instead of a "gut-brain axis", it would be more correct to refer to the "GM-gut-brain axis" integrating the GM with neuro-humoral signals from/to the CNS, neuroendocrine and immune systems, the autonomic nervous system, and the enteric nervous system (ENS). A growing amount of evidence has pinpointed the availability and metabolism of the essential amino acid tryptophan as a key regulator of this axis. Tryptophan is metabolized along the serotonin or the kynurenine pathway [86] with many implications for ENS and CNS functioning (Fig. 1). Serotonin is mainly $(95 \%)$ located within the GI tract and in a small proportion (5\%) in the CNS. In the gastrointestinal tract serotonin is responsible for motility, secretion and absorption as well as intestinal transit, while it can also modulate food intake by stimulating vagal afferent pathways involved in the reduction of obesity and metabolic dysfunction [87]. By contrast, most available tryptophan is transformed into quinolic and kynurenic acid, which are of particular interest for neurogastroenterology as they are neuroactive metabolites that act on $N$-methyl-D-aspartate (NMDA) and alpha 7 nicotinic acetylcholine receptors in the CNS and ENS. In the CNS, kynurenic acid has long been viewed as neuroprotective, whilst quinolinic acid is primarily considered an excitotoxic NMDA receptor agonist [88]. Within the gastrointestinal tract, both molecules appear to be involved in immunoregulation [89] and in particular kynurenic acid may have anti-inflammatory properties [90]. Due to its specific role on tryptophan metabolism and serotonergic system, there is some evidence that the GM is a pivotal player in the regulation of different behavioral domains such as pain, depression, anxiety and cognition [86]. Major studies about this relationship have been performed using germfree animals (free of all microorganisms, including those normally symbiotic in the gut) characterized by increased plasma tryptophan concentrations that can be normalized by colonizing the mice immediately post-weaning. These animals exhibited increased hippocampal 5-hydroxytryptophan (a serotonin precursor) concentration and significant CNS alterations, demonstrating that the GM is essential for normal brain development [91, 92]. In fact, the GM can 


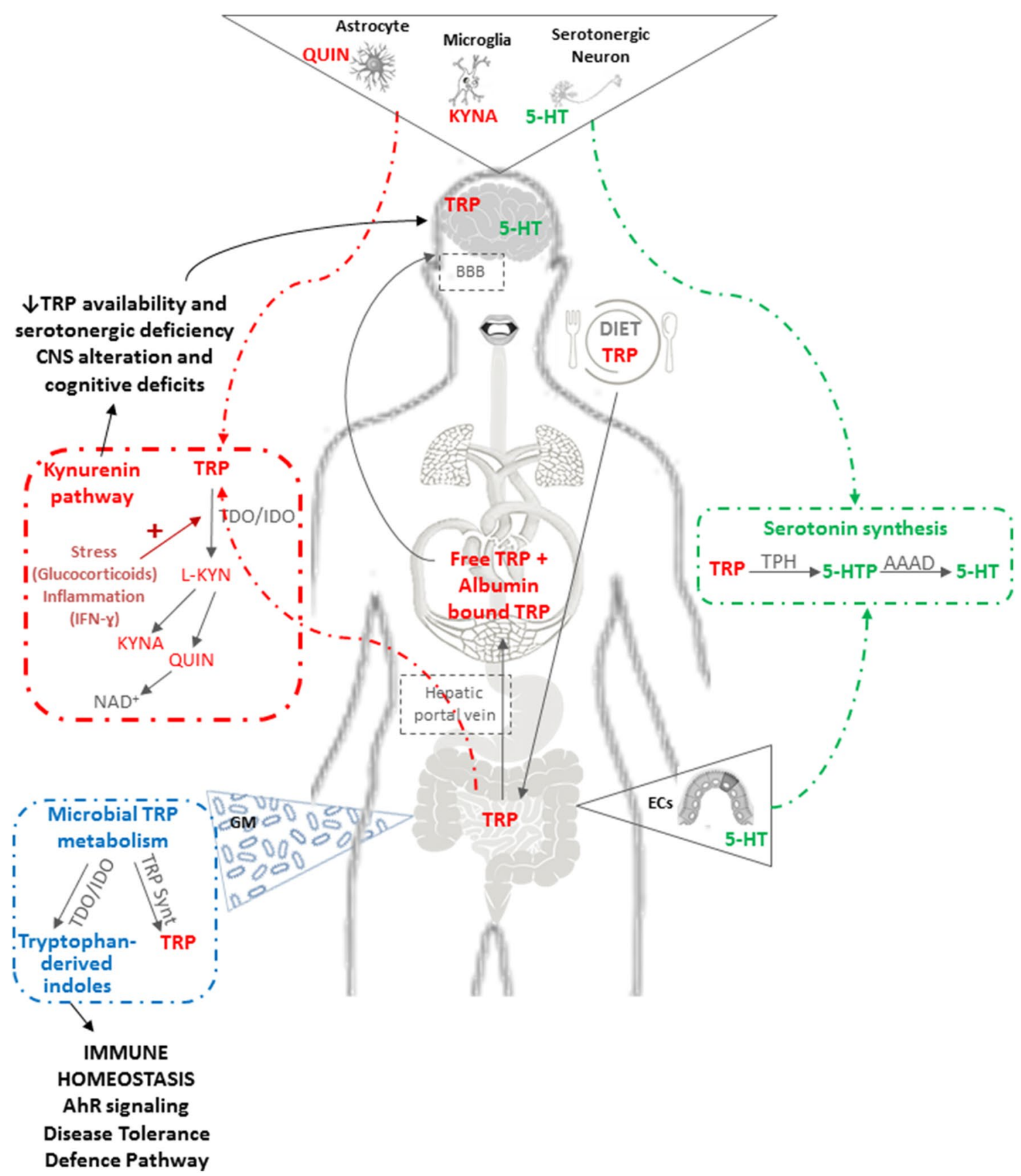

directly utilize tryptophan, limiting its availability to the host because bacteria require tryptophan for their normal growth and some strains such as Bacteroides fragilis may produce a tryptophanase, an enzyme that has recently been associated with autism spectrum disorders [93] (Fig. 1). Moreover, some bacterial strains are able to synthesize tryptophan and produce serotonin from tryptophan in vitro. Tryptophan, through the kynurenine pathway, is involved in the biosynthesis of nicotinamide adenine dinucleotide $\left(\mathrm{NAD}^{+}\right)$[94], which has a key role in human health as it is an essential coenzyme for the cellular processes of energy metabolism, cell protection and biosynthesis. Moreover, decreased cellular $\mathrm{NAD}^{+}$concentrations occur during aging and supplementation with $\mathrm{NAD}^{+}$precursors can prolong both life span and health span $[95,96]$. NAD ${ }^{+}$is indeed an important co-substrate of sirtuins. Several papers have shown that in old animals, when the levels of $\mathrm{NAD}^{+}$are restored, there is an increase in sirtuin 1 and a reduction in mitochondrial stress, DNA damage and inflammation [95].

Tryptophan-derived indoles are involved in the host-microbiome interaction in the intestine [97]. Indoleamine-2,3-di-oxygenase (IDO) is an interferon$\gamma$-induced enzyme involved in catabolizing tryptophan to kynurenine, which has been shown to be higher in 
\Fig. 1 Tryptophan metabolism through the serotonin and kynurenine pathway. Tryptophan (TRP) is an essential amino acid which must be supplied with the diet. Once absorbed from the gut, TRP is made available in circulation as free TRP and albumin-bound TRP fraction and/or is metabolized along the serotonin or the kynurenine pathway. TRP in circulation can cross the blood-brain barrier (BBB) to participate in serotonin (5-HT) synthesis in the CNS. TRP in the gut is metabolized to 5-HT in the enterochromaffin cells (ECs): TRP is first converted to 5-hydroxytryptophan (5-HTP) by the rate-limiting enzyme tryptophan hydroxylase (TPH), then the short-lived 5-HTP intermediate product is decarboxylated to 5-HT by aromatic amino acid decarboxylase (AAAD). However, the vast majority of available TRP is metabolized along the kynurenine pathway. Kynurenine (L-KYN) is produced from TRP by the action of the hepatic enzyme, tryptophan-2,3-dioxygenase (TDO) or the ubiquitous indoleamine2,3-dioxygenase (IDO). TDO can be induced by glucocorticoids or by TRP itself, whereas IDO is stimulated by inflammation with IFN- 8 as the most potent inducer. Once L-KYN is produced, it is further metabolized along one of two distinct arms of the pathway with the production of neuroprotective kynurenic acid (KYNA) or neurotoxic quinolinic acid (QUIN). KYNA can be neuroprotective against QUIN-induced excitotoxicity but it can also induce cognitive impairment when abnormally elevated. Activation of the kynurenin pathway has a dual impact by limiting the availability of TRP for 5-HT synthesis and increasing the downstream production of neurotoxic/ neuroprotective metabolites. TRP, via the kynurenine pathway, is involved in the biosynthesis of nicotinamide adenine dinucleotide $\left(\mathrm{NAD}^{+}\right)$which is an essential coenzyme for cellular processes of energy metabolism, cell protection and biosynthesis. The GM can also directly utilize TRP, limiting its availability to the host. Certain bacterial strains may produce a tryptophanase enzyme that synthetizes indoles from TRP. These microbial metabolites have recently been identified as human aryl hydrocarbon receptor (AhR)-selective agonists. AhR signaling has a role in chemical/microbial defense and tissue development, while, recently, IDO-AhR axis has been recognized as a fundamental player in controlling the "Disease Tolerance Defense Pathway". Bacteria can also synthesize tryptophan via enzymes such as TRP synthase (TRP synt) and specific bacterial strains can also produce serotonin from TRP in vitro. The balance between bacterial TRP utilization and metabolism, TRP synthesis and 5-HT production plays an important role in regulating gastrointestinal and circulating TRP availability for the host in addition to its dietary intake. Moreover, accumulating evidence supports the role of the GM in regulating TRP availability and 5-HT synthesis via modulation of the enzymes responsible for TRP degradation along the kynurenine pathway

nonagenarians than in young people [98]. Hence, inflammaging might induce IDO, leading to tryptophan degradation to kynurenine. Microbial tryptophan metabolites generated by induction of IDO have recently been identified as human aryl hydrocarbon receptor (AhR)-selective agonists [99]. AhR signaling has a role in various physiological processes including chemical/microbial defence and tissue development, while, recently, the IDO-AhR axis has been recognized as a fundamental player in the control of the "Disease Tolerance Defence Pathway", i.e., the ability of the host to reduce the effect of infection on host fitness [100] (Fig. 1). Data obtained on murine models have shown that tryptophan catabolism by IDO assumes an immunoregulatory role acting via AhR ligands, boosting regulatory $\mathrm{T}$ cells and protecting mice from chronic hyperinflammatory responses [101].

The balance between bacterial tryptophan utilization, metabolism and synthesis and serotonin/kynurenine production has a fundamental function in determining local gastrointestinal and circulating tryptophan availability for the host with implications for both ENS and CNS serotonergic neurotransmission [86].

Modifications to the composition of GM across the lifespan may deeply affect the availability of tryptophan and serotonergic signaling during aging. Shotgun analysis on the bacterial metagenome of young, old and centenarian subjects showed an age-related amplified abundance of genes involved in the tryptophan metabolism pathway [102] and this finding is in agreement with the reduction of tryptophan due to its altered bio-availability found in the serum of centenarians [7, 71]. Little is known about plasma tryptophan disposition in aged experimental animals, while in humans the plasma concentration of tryptophan is moderately lower in the elderly [103]. It is interesting to note that in rodents, limiting the dietary intake of tryptophan and methionine may have a beneficial effect on health- and life span [104], while excess of tryptophan can be toxic and carcinogenic [105]. In addition, alteration of the kynurenine metabolites may contribute to neurotoxicity [106] and has been associated with Huntington disease [107], HIV dementia [108] and Parkinson's disease (PD) [109]. Surprisingly, in a mouse model of Alzheimer's disease (AD), a diet rich in tryptophan seems able to reduce the amyloid plaque content [110]. It is thus tempting to speculate that the GM of centenarians adjusted the tryptophan metabolism to support healthy aging (Fig. 2). These findings assume a particular importance in view of the fact that centenarians are remarkably free from neurodegenerative pathologies such as PD and AD. Indeed, although the prevalence of cognitive impairment in centenarian studies varies widely [111], some of them (15-20\%) preserve cognitive function and, even among those who show cognitive impairment at 100 years, approximately $90 \%$ delay the onset of clinically evident dementia until the advanced average age of 92 years [112]. In addition, most centenarians have low levels of anxiety and depression [111], suggesting that such people should be chosen as "super-controls" in studies designed to evaluate the contribution of GM dysbiosis to cerebral degenerative diseases.

$\mathrm{AD}$ is one of the commonest neurodegenerative disorders and associates with cerebral accumulation of amyloidbeta fibrils driving neuroinflammation and neurodegeneration. The bacterial species residing in the intestine have been shown to release substantial amounts of amyloids and lipopolysaccharides, thereby promoting the production of pro-inflammatory cytokines and modulating the signaling pathways involved in the pathogenesis of $\operatorname{AD}[113,114]$. Numerous research findings have shown that AD may start 


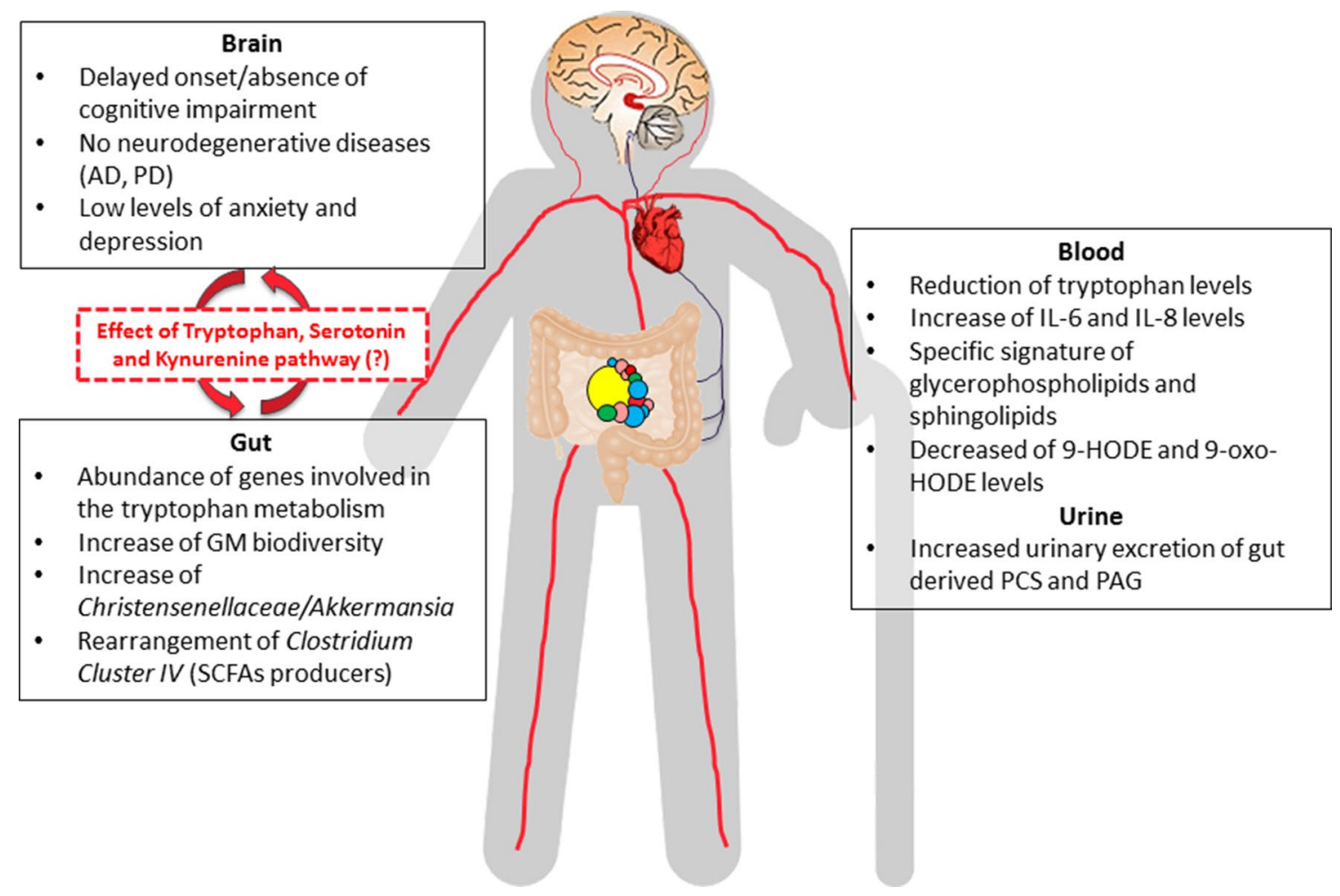

Fig. 2 Gut microbiota and brain function in Italian centenarians. This figure summarizes our studies on the phenotypic characteristics of Italian centenarians. In extreme longevity complex remodeling of the GM is reflected at a systemic level by specific signatures of blood and urine markers (inflammatory, lipidic and metabolic). The strong two-way connection between GM and brain is likely to positively affect the well-preserved cognitive function of centenarians until a

in the gut, and hence is closely associated with GM imbalance. There is increasing evidence to suggest a link between GM and PD. Recent studies showed that PD is associated with gut dysbiosis [115]; the fecal concentration of SCFAs is significantly reduced in PD patients compared to controls and this reduction could impact on CNS alterations and contribute to gastrointestinal dysmobility in PD [116]. In a mouse model of PD, it has been demonstrated that GM is a key player in motor deficits and microglia activation [117]. Interestingly, alpha-synuclein aggregates, a pivotal marker of PD, are present in both the submucosal and myenteric plexuses of the ENS, prior to their appearance in the brain, indicating a possible gut to brain route of "prion-like" spread [118].

The GM role has also been investigated regarding regulation of hypothalamic-pituitary-adrenal (HPA) axis development [119, 120]. In germ-free mice, exposure to a restraint stress triggers an exaggerated HPA axis response, as compared to specific pathogen-free control mice. Such an aberrant response is normalized through intestinal colonization by Bifidobacterium longum subsp. infantis, and fecal matter from specific pathogen-free mice. Importantly, fecal very advanced age. The fundamental role in the effect on the brain by bacterial tryptophan metabolism via the serotonin and/or kynurenine pathways deserves to be further investigated. $A D$ Alzheimer's disease, $P D$ Parkinson's disease, $S C F A s$ short-chain fatty acids, $I L-6$ interleukin-6, IL-8 interleukin-8, 9-HODE 9-hydroxy-octadecadienoic acid, 9-oxo-HODE 9-oxo-octadecadienoic acid, PCS p-cresol sulfate, $P A G$ phenylacetylglutamine

microbiota transplantation (FMT) proved efficient only in animals' early life [121]. These experiments demonstrated the crucial function of the GM in the development of an appropriate physiological endocrine response versus stress in the postnatal stage of the animal model. During life, chronic HPA axis hyperactivation by stress exposure damages the gut barrier integrity, causing intestinal dysbiosis, behavioral changes and stress-related symptoms, including mood disorders, anxiety and cognitive defects [122]. Patients suffering from hepatic encephalopathy are characterized by alterations of GM composition and endotoxemia. In particular, high levels of inflammatory cytokines were found in cirrhotic patients with cognitive decline, as compared to those with normal cognitive function, and the bacterial families Alcaligeneceae and Porphyromonadaceae proved positively correlated with cognitive impairment [123, 124]. Other works have focused on the impact of the GM on depression or anxiety, showing that pathogen-free mice exhibit reduced anxiety and increased motor activity [91, 125]. Tillisch et al. [126] demonstrated that brain activity and connectivity in healthy women following an emotive task could be attenuated by administering a 4 -week course of a fermented 
milk beverage containing several probiotic bacterial strains [126]. Thus, the GM seems to modulate multiple effects, overcoming even the adaptive immunity functions besides revealing neurological/psychological potential. The two-way interaction between GM and the brain can be modulated by diet and/or probiotic/prebiotic/symbiotic supplementation designed to positively impact on brain activity and behavior [127]. For these reasons, probiotics with psychotropic functions in humans, such as Lactobacillus helveticus and Bifidobacterium longum, have recently been termed "psychobiotics" given their ability to reverse anxiety or depression-like behavior [128].

\section{Gut microbiota-targeted diets and interventions improving cognition and health}

The marked potential effect of the GM on neurological and psychological pathways suggested the hypothesis that intestinal bacteria may be a bridge in the emerging relation between diet and the cognitive system [123]. For example, pronounced consumption of fruit, vegetables and pulses typical of the Mediterranean Diet (MedDiet) has been associated with increasing fecal SCFA levels. SCFAs (acetate, propionate and butyrate), produced by GM (Firmicutes and Bacteroidetes strains) during fermentation of undigested polysaccharides, has a well-documented protective role on various inflammatory as well as behavioral disorders [129, 130].

It has recently been shown that the GM rapidly responds to altered diet in a diet-specific manner. It seems possible to modulate GM composition and activity within a single day, switching from herbivorous to carnivorous diet and as a consequence modulating GM metabolic pathways [131]. Thus, the dietary lifestyle represents a long-life stimulus for the GM, which responds by modifying its structure and functionality in the short term with multiple effects on the organism.

Recently, it has been postulated that the MedDiet exerts its health effects through hormetic mechanisms [132]. A lifelong exposure to the specific components of the MedDiet may, therefore, very likely counteract the effects of inflammatory stimuli, including those that may come from the GM metabolism, by acting as hormetins [132]. Epidemiologic evidence also suggests that coffee drinkers have a lower risk of PD [133]. It has been proposed that this protective effect impacts on the composition of the GM, counteracting the development of intestinal inflammation which is associated with less misfolding of the protein alpha-synuclein in the enteric nerves. This would reduce the risk of PD development, minimizing propagation of the alpha-synuclein aggregates to the CNS [118].

In animal models, interventions aimed at reducing calorie intake have been shown to be accompanied by structural modulation of the GM [134]. For instance, a life-long lowfat diet significantly altered the overall structure of the GM in C57BL/6J mice. Calorie restriction was shown to enrich phylotypes positively correlating with longevity, such as the genus Lactobacillus, and to reduce phylotypes negatively associated with lifespan [135]. Since nutrient metabolism is highly dependent on the composition of the GM and vice versa [136], it can be assumed that certain anti-aging interventions may cause specific variations to gut microbial communities causing chronic calorie restriction conditions and thus promoting both the health span and the life span. Several documented clinical trials have investigated the effect of prebiotics and probiotics, particularly those containing Bifidobacterium and Lactobacillus, as a microbiota-targeted intervention to improve health status in elderly populations [137-140]. Most of the benefits are mediated by the activation of anti-inflammatory pathways in the residents' microorganisms. Probiotic supplementation may also improve metabolic and cardiovascular health status [141] and promote longevity by stimulating the innate immune response [142, 143], improving resistance to oxidative stress [144], decreasing lipofuscin accumulation [145] and modulating serotonin signaling [146]. There is also evidence that probiotic treatment can promote longevity in mice, possibly through suppression of chronic low-grade inflammatory processes in the colon [147]. Importantly, several findings suggest that direct modulation of the GM may not only be applied in treating particular age-related disorders, but can also be a promising therapeutic option to combat the aging process per se. For example, in a murine model, oral administration of purified exopolysaccharide fractions from Bifidobacterium animalis $\mathrm{RH}$ that were isolated from the fecal samples of centenarians residing in Bama longevity villages (Guangxi, China) resulted in significantly increased activity by superoxide dismutase, catalase and total antioxidant capability in serum, as well as reduced levels of lipofuscin accumulation in the mouse brain [148].

Another approach to restoring the intestinal ecosystem is FMT, also called bacteriotherapy, a transfer of liquid filtrate feces from a healthy donor into the recipients' gastrointestinal tract to treat a particular disease or condition [149]. Initially, bacteriotherapy was developed as an effective method of treating Clostridium difficile infection, which is a major cause of healthcare-associated diarrhea through perturbation of the normal GM [150]. More recently, its potential effectiveness and safety has been hypothesized in the prevention and treatment of non-gastrointestinal pathologic conditions, including those commonly associated with aging, e.g., atherosclerosis, metabolic syndrome, type 2 diabetes and neurodegenerative diseases $[151,152]$. In a preliminary study of the effectiveness of FMT in humans, transferring GM from lean donors to persons with metabolic syndrome [153] beneficially affected the GM composition in recipients 
by increasing amounts of butyrate-producing bacteria along with improved insulin sensitivity 6 weeks after the FMT procedure [154]. Improvements in symptoms of PD in patients receiving FMT were described in one case report [155], while no studies have been reported for $\mathrm{AD}$ so far.

In this scenario the knowledge emerging from GM studies in centenarians may soon be exploited for therapeutic purposes. For example, transplantation of centenarians' GM into germ-free animal models will allow us to identify the bacteria or bacterium combination that could be protective against neurodegenerative diseases.

\section{Gut microbiota and host genetics: an intimate evolutionary-shaped relationship}

During the last few years, an impressive amount of literature has been published on the different strategies to modify and improve the GM diversity structure with a view to promoting human health. Similarly, many pathologies ranging from obesity and inflammatory diseases to behavioral and physiological abnormalities with neurodevelopmental disorders have been associated with different types of bacterial species and their products [77], as described in the previous sections.

On the other hand, recent data have suggested a new and intriguing possibility that the host genome interacts and shapes its own GM. In this connection host genetics have been shown to influence the composition of the GM in twin studies [156, 157], while more recently in a wider population study, Christensenellaceae have been reported as the chief bacterium family associated with genetics [158]. The abundance of Christensenellaceae was also associated with lower body mass index (BMI) in twins, and when introduced into a mouse model it led to reduced weight gain in treated mice compared with controls [158], suggesting that the microbiome can be an important mediator between host genetics and phenotype. Intriguingly, these bacteria were found to characterize the GM in extreme longevity [8], thus reinforcing the idea of a close association with the genetic background and suggesting a possible link to the inheritable component of human longevity. Nuclear, also mitochondrial, DNA plays a major role in the aging process so the complex interaction between these two host genetics [159] should be taken into account if we are to properly address the GM remodeling occurring during the human life span.

The intimate symbiotic relationship between host genetics and the GM is very ancient since vertebrates coevolved along with their gut bacteria. Multiple lineages of the predominant bacterial taxa such as Bacteroidaceae and Bifdobacteriaceae in the gut arose via co-speciation within hominids over the past 15 million years [160]. The divergence times also indicate that nuclear, mitochondrial, and gut bacterial genomes diversified in concert during hominid evolution [160]. Interestingly, it seems that gut microbiomes have recorded the information of major dietary shifts that occurred during the evolution of mammals, allowing us to predict ancient diets from the reconstruction of ancient microbiomes [161].

Recently, genome-wide association screening for host genetic associations with GM composition identified 42 loci (mainly related to innate immunity) associated with GM variation and function in humans [162]. Another study identified significant associations between gut microbial characteristics and the VDR gene (encoding vitamin D receptor), in addition to a large number of other host genetic factors, and eventually quantified the total contribution of host genetic loci to diversity as $10.43 \%$ [163]. The non-genetic factors such as age, sex, BMI, smoking status and dietary patterns explain $8.87 \%$ of the observed variations in the GM [163]. Even though the effect of individual genes is small and comparable with the cumulative effect of key non-genetic covariates, the underlying biology of these studies provides a critical framework for future assessments of host-microbe interactions in humans with an adequate statistical power and sample size. Associations with gut microbial community composition at the VDR locus provide a link with secondary bile acids, which serve as ligands for VDR. Results from gene set enrichment analysis and the observation that the bile acid profile in serum associates with variation in the gut microbiome [163] further support this finding. A detailed description of the effect of host genetics on GM composition lies outside the scope of this review. Kurilshikov and colleagues recently published a comprehensive summary of the state of the art on host genetic determinants of GM with details as to techniques and populations analyzed, to which readers are referred [164].

A recent bioinformatics analysis predicts that long noncoding RNAs expressed in the intestinal epithelial cell in murine models constitute molecular signatures reflecting the different types of microbiome [165]. In this direction, very recent data highlight the role of the host genome in shaping the GM, even if in terms of microRNAs (miRs). MiRs produced by gut epithelial cells enter bacterial membrane, modifying bacterial gene expression in in vitro models [166]. In a mouse model (DICER deficiency), a severe dysbiosis develops when miR maturation is deficient. These important findings not only outline the tight coevolution and inter-organismal crosstalk leading to various profound cellular and metabolic changes, but also lay the foundations for new miR-based therapies to counteract gut-related diseases.

Many variables may be responsible for GM remodeling associated with human longevity. Among these, the genetic makeup of extreme longevity [159, 167], and the epigenetic changes associated with aging could have a deep impact together with nutrition and lifestyle habits. These lifelong 
interactions by variables are expected to have significant outputs in the production of specific blood/urine biomarkers or longevity-associated metabotypes. This is the case with centenarians. As reported above, Italian centenarians show increased excretion of bacterial products such as PAG and PCS in urine [7], specific blood lipid profiles and changes in amino acid levels [7, 71] (Fig. 2). By contrast, centenarians from the Bama County in China showed decreased levels of PCS and increased levels of fecal SCFAs and total bile acids [73]. All these findings support the hypothesis of a complex remodeling of the lipid and amino acid metabolism correlated with GM changes [7], as a result of lifelong adaptation and coevolution processes that could also be ethnic specific. Of note, it still remains to be clarified what role gender plays in GM modification studies on long-lived subjects, since female centenarians outnumber males. A much deeper knowledge of the relationship between host genetics and the GM emerged from a recent paper, which used shotgun analysis on 250 adult twins from the UK [168]. These data showed that GM composition and functions are inheritable and that twin pairs share microbial SNPs. Interestingly, this similarity is lost after decades of living apart [168], emphasizing the impact of household and geographic region on the GM.

\section{Lifelong interaction among sex, sex hormones and gut microbiota}

Several studies have shown that sex hormones also play a role in the host-microbiota interaction. Indeed the term "microgenderome" defines the potential mediating and modulatory role of sex hormones on GM function and composition with implications for autoimmune and neuroimmune conditions [169]. Sexual dimorphism is common in autoimmune diseases. Using the non-obese diabetic mouse model of Type 1 Diabetes, Markle et al. showed that the gut commensal microbial community strongly conditions the pronounced sex bias in Type 1 Diabetes risk by controlling serum testosterone and metabolic phenotypes [170]. Their results revealed evidence of sex-specific microbial communities and sex-specific responses to the same microbial communities. The same group also found that the recipients' GM was stably altered in a sex-specific way, since maletypical changes in the GM of female recipients were evident for several months. Unexpectedly, these experimental GM manipulations strongly protected the female mice from diabetes. The mechanism behind this protection critically depended on the impact of the GM on host metabolism and sex hormone signaling pathways [171]. A number of different taxa have been found between male and female mice, while the sex differences in GM composition depend in part on genetic background [172]. Using gonadectomized and hormone-treated mice clearly revealed hormonal effects on the GM composition [172]. In humans, sex-specific interactions between Firmicutes and neurological, immune and mood symptoms of myalgic encephalomyelitis/chronic fatigue syndrome have been reported [173], but we are just beginning to appreciate the links between human microbiome composition and hormonal phenotypes. Twin studies have revealed that the once similar microbial composition of opposite-sex twins becomes distinctly different after puberty when compared to that of same-sex twins which remains compositionally similar [57]. These data suggest that agespecific interactions of the host with specific microbes may exert beneficial and/or detrimental influences on the biology of the host, including either protection from or susceptibility to autoimmune disease. Furthermore, microbiota transfer studies in humans, mice, and rats reveal a high degree of host specificity on the part of the GM. Bacterial gene expression modulation by the host may partly explain the failure of FMT in certain specific cases, such as those related to Clostridium difficile infection treatment [174] and eventually impact on GM remodeling with age [8]. Efficient colonization and associated effects also seem to be most successful in young animals, most likely because their microbiota is not yet stabilized [169].

Dietary effects on the composition and diversity of GM depend in part on sex-specific interactions [172, 175]. An interesting work showed that GM composition depends on interactions between host diet and sex within populations of wild and laboratory fish, laboratory mice and humans. The inter-individual diet variation correlates with individual differences in the GM and these diet-microbiota associations are sex dependent. In mice, experimental diet manipulations confirmed that diet affects the GM differently in males versus females. Thus, the prevalence of the individual genotype interacting with the environment (e.g., sex by diet) implies that therapies to treat dysbiosis might have sex-specific effects [176].

\section{Conclusions}

Overall, the data available on lifelong changes in the GM are still too few for us to draw any definitive conclusions as to the basic question of how much can be set down to variables such as population, diet, genetics and gender, and how much to the aging process per se. In particular, the GM changes occurring in the last two or three decades of life (in nonagenarians, centenarians, semi-supercentenarians and supercentenarians, i.e., persons who reach the age of 110 years) have been insufficiently investigated, especially regarding the possible contribution of GM to health and longevity or to cognitive decline and neurodegeneration. Longitudinal studies envisaging metagenomics sequencing and in-depth phylogenetic analysis as well as an extensive phenotypic 
characterization using up-to-date omics (metabolomics, transcriptomics and meta-transcriptomics, to mention a few) are urgently needed. The results of this comprehensive approach are likely to offer more satisfactory answers to the questions addressed in this paper.

Acknowledgements This work was supported by the Italian Ministry of Health "Ricerca Finalizzata" young Researchers (under 40)/Giovani Ricercatori No. GR-2013-02,358,026) to A.S., the European Union (EU)'s H2020 Project 'Propag-ageing' (Grant Agreement No. 634821), the EU JPND 'Adage', the EU FP7 NU-AGE (Grant Agreement No. 266486) to C.F. and the University of Bologna FARB linea 2 Project 2014 No. RFBO120790 to M.C.

\section{Compliance with ethical standards}

Conflict of interest The authors have no relevant interests to declare.

Open Access This article is distributed under the terms of the Creative Commons Attribution 4.0 International License (http://creativecommons.org/licenses/by/4.0/), which permits unrestricted use, distribution, and reproduction in any medium, provided you give appropriate credit to the original author(s) and the source, provide a link to the Creative Commons license, and indicate if changes were made.

\section{References}

1. Biagi E, Rampelli $\mathrm{S}$, Turroni $\mathrm{S}$ et al (2016) The gut microbiota of centenarians: signatures of longevity in the gut microbiota profile. Mech Ageing Dev. doi:10.1016/j.mad.2016.12.013

2. Candela M, Biagi E, Turroni S et al (2015) Dynamic efficiency of the human intestinal microbiota. Crit Rev Microbiol 41:165-171. doi:10.3109/1040841X.2013.813900

3. Rampelli S, Candela M, Turroni S et al (2015) Microbiota and lifestyle interactions through the lifespan. Trends Food Sci Technol 57:265-272. doi:10.1016/j.tifs.2016.03.003

4. Garagnani P, Giuliani C, Pirazzini C et al (2013) Centenarians as super-controls to assess the biological relevance of genetic risk factors for common age-related diseases: a proof of principle on type 2 diabetes. Aging (Albany NY) 5:373-385

5. Fortney K, Dobriban E, Garagnani P et al (2015) Genome-wide scan informed by age-related disease identifies loci for exceptional human longevity. PLoS Genet 11:e1005728. doi:10.1371/ journal.pgen.1005728

6. Horvath S, Pirazzini C, Bacalini MG et al (2015) Decreased epigenetic age of PBMCs from Italian semi-supercentenarians and their offspring. Aging (Albany NY) 7:1159-1170. doi:10.18632/ aging. 100861

7. Collino S, Montoliu I, Martin FPJ et al (2013) Metabolic signatures of extreme longevity in northern italian centenarians reveal a complex remodeling of lipids, amino acids, and gut microbiota metabolism. PLoS One 8(3):e56564. doi:10.1371/journal. pone.0056564

8. Biagi E, Franceschi C, Rampelli S et al (2016) Gut microbiota and extreme longevity. Curr Biol. doi:10.1016/j.cub.2016.04.016

9. Giuliani C, Barbieri C, Li M et al (2014) Transmission from centenarians to their offspring of mtDNA heteroplasmy revealed by ultra-deep sequencing. Aging (Albany NY) 6:454-467. doi:10.18632/aging. 100661
10. Franceschi C, Bonafè M (2003) Centenarians as a model for healthy aging. Biochem Soc Trans 31:457-461. doi:10.1042/ BST0310457

11. Kheirbek RE, Fokar A, Shara N et al (2017) Characteristics and incidence of chronic illness in community-dwelling predominantly male US veteran centenarians. J Am Geriatr Soc. doi:10.1111/jgs.14900

12. Perls T, Kunkel LM, Puca AA (2002) The genetics of human longevity. J Am Geriatr Soc 50:359-368. doi:10.1046/j.1532-5415.2002.49283.x

13. Schoenmaker M, Jm De Craen A, Hem De Meijer P et al (2006) Evidence of genetic enrichment for exceptional survival using a family approach: the Leiden Longevity Study. Eur J Hum Genet 14:79-84. doi:10.1038/sj.ejhg.5201508

14. Bucci L, Ostan R, Cevenini E et al (2016) Centenarians' offspring as a model of healthy aging: a reappraisal of the data on Italian subjects and a comprehensive overview. Aging (Albany NY) 8:1-11. doi:10.18632/aging.100912

15. Terry DF, Wilcox ÃMA, Mccormick MA et al (2004) Lower all-cause, cardiovascular, and cancer mortality in centenarians' offspring. JAGS 52:2074-2076. doi:10.1111/j.1532-5415.2004.52561.x

16. Atzmon G, Schechter C, Greiner W et al (2004) Clinical phenotype of families with longevity. J Am Geriatr Soc 52:274-277

17. Ikeda $\mathrm{A}$, Iso $\mathrm{H}$, Toyoshima $\mathrm{H}$ et al (2006) Parental longevity and mortality amongst Japanese men and women: the JACC Study. J Intern Med 259:285-295. doi:10.1111/j.1365-2796.2005.01609.x

18. Caselli G, Pozzi L, Vaupel JW et al (2006) Family clustering in Sardinian longevity: a genealogical approach. Exp Gerontol. doi:10.1016/j.exger.2006.05.009

19. Ostan R, Bucci L, Capri M et al (2008) Immunosenescence and immunogenetics of human longevity. Neuroimmunomodulation 15:224-240. doi:10.1159/000156466

20. Monti D, Ostan R, Borelli V et al (2017) Inflammaging and human longevity in the omics era. Mech Ageing Dev. doi:10.1016/j.mad.2016.12.008

21. Franceschi $C$, Valensin $S$, Bonafè $M$ et al (2000) The network and the remodeling theories of aging: historical background and new perspectives. Exp Gerontol 35:879-896

22. Franceschi C, Monti D, Sansoni P, Cossarizza A (1995) The immunology of exceptional individuals: the lesson of centenarians. Immunol Today 16:12-16

23. Franceschi C, Bonafè $M$, Valensin $S$ et al (2000) Inflamm-aging. An evolutionary perspective on immunosenescence. Ann N Y Acad Sci 908:244-254. doi:10.1111/j.1749-6632.2000.tb06651.x

24. Franceschi C, Capri M, Monti D et al (2007) Inflammaging and anti-inflammaging: a systemic perspective on aging and longevity emerged from studies in humans. Mech Ageing Dev 128:92105. doi:10.1016/j.mad.2006.11.016

25. Ginaldi L, Demartinis M, Monti D, Franceschi C (2005) Chronic antigenic load and apoptosis in immunosenescence. Trends Immunol 26:79-84. doi:10.1016/j.it.2004.11.005

26. Franceschi C, Garagnani P, Vitale $G$ et al (2017) Inflammaging and "Garb-aging". Trends Endocrinol Metab 28:199-212. doi:10.1016/j.tem.2016.09.005

27. Christensen K, Thinggaard M, Oksuzyan A et al (2013) Physical and cognitive functioning of people older than 90 years: a comparison of two Danish cohorts born 10 years apart. Lancet 382:1507-1513. doi:10.1016/S0140-6736(13)60777-1

28. Department of Economic and Social Affairs, Population Division (2015) World population prospects: The 2015 revision. Key findings and advance tables. Working paper no. ESA/P/WP.241. United Nation, New York

29. Collado MC, Rautava S, Aakko J et al (2016) Human gut colonisation may be initiated in utero by distinct microbial communities 
in the placenta and amniotic fluid. Sci Rep 6:23129. doi:10.1038/ srep23129

30. Isolauri E, Sherman PM, Walker WA (eds) (2017) Intestinal microbiome: Functional aspects in health and disease. Nestlé nutrition institute workshop sries, vol 88. Nestec Ltd., Vevey/S. Karger AG, Basel, pp 11-21. doi:10.1159/000455209

31. Douwes J, Cheng S, Travier N et al (2008) Farm exposure in utero may protect against asthma, hay fever and eczema. Eur Respir J 32:603-611. doi:10.1183/09031936.00033707

32. Conrad ML, Ferstl R, Teich R et al (2009) Maternal TLR signaling is required for prenatal asthma protection by the nonpathogenic microbe Acinetobacter lwoffii F78. J Exp Med 206:28692877. doi:10.1084/jem.20090845

33. Rutayisire E, Huang K, Liu Y, Tao F (2016) The mode of delivery affects the diversity and colonization pattern of the gut microbiota during the first year of infants' life: a systematic review. BMC Gastroenterol 16:86. doi:10.1186/s12876-016-0498-0

34. Edwards CA (2017) Determinants and duration of impact of early Gut bacterial colonization. Ann Nutr Metab. doi:10.1159/000466711

35. Lee YK, Mazmanian SK (2010) Has the microbiota played a critical role in the evolution of the adaptive immune system? Sci (Washington, DC) 330:1768-1773. doi:10.1126/science.1195568.Has

36. Biagi E, Candela M, Fairweather-Tait S et al (2012) Aging of the human metaorganism: the microbial counterpart. Age (Dordr) 34:247-267. doi:10.1007/s11357-011-9217-5

37. Clarke G, Stilling RM, Kennedy PJ et al (2014) Minireview: gut microbiota: the neglected endocrine organ. Mol Endocrinol 28:1221-1238. doi:10.1210/me.2014-1108

38. Rajilić-Stojanović M, de Vos WM (2014) The first 1000 cultured species of the human gastrointestinal microbiota. FEMS Microbiol Rev 38:996-1047. doi:10.1111/1574-6976.12075

39. Eckburg PB, Bik EM, Bernstein CN et al (2005) Diversity of the human intestinal microbial flora. Science (80-) 308:1635-1638. doi:10.1126/science.1110591

40. Lozupone CA, Stombaugh JI, Gordon JI, Jansson JK, Knight R (2012) Diversity, stability and resilience of the human gut microbiota. Nature 489:220-230. doi:10.1038/nature11550

41. Candela M, Biagi E, Brigidi P et al (2014) Maintenance of a healthy trajectory of the intestinal microbiome during aging: a dietary approach. Mech Ageing Dev 136-137:70-75. doi:10.1016/j.mad.2013.12.004

42. Claesson MJ, Jeffery IB, Conde S et al (2016) Gut microbiota composition correlates with diet and health in the elderly. Nature 488:178-184. doi:10.1038/nature11319

43. Claesson MJ, Cusack S, O'Sullivan O et al (2011) Composition, variability, and temporal stability of the intestinal microbiota of the elderly. Proc Natl Acad Sci 108:4586-4591. doi:10.1073/ pnas. 1000097107

44. Round JL, Mazmanian SK (2009) The gut microbiota shapes intestinal immune responses during health and disease. Nat Rev Immunol 9:313-323. doi:10.1038/nri2515

45. Guigoz Y, Doré J, Schiffrin EJ (2008) The inflammatory status of old age can be nurtured from the intestinal environment. Curr Opin Clin Nutr Metab Care 11:13-20. doi:10.1097/ MCO.0b013e3282f2bfdf

46. Woodmansey EJ (2007) Intestinal bacteria and ageing. J Appl Microbiol 102:1178-1186. doi:10.1111/j.1365-2672.2007.03400.x

47. Buford TW (2017) (Dis)Trust your gut: the gut microbiome in age-related inflammation, health, and disease. Microbiome 5(1):80. doi:10.1186/s40168-017-0296-0

48. Jackson M, Jeffery IB, Beaumont M et al (2016) Signatures of early frailty in the gut microbiota. Genome Med 8:8. doi:10.1186/ s13073-016-0262-7
49. Thevaranjan N, Puchta A, Schulz C et al (2017) Age-associated microbial dysbiosis promotes intestinal permeability, systemic inflammation, and macrophage dysfunction. Cell Host Microbe 21(455-466):e4. doi:10.1016/j.chom.2017.03.002

50. O'Toole PW, Jeffery IB (2015) Gut microbiota and aging. Science 350(6265):1214-1216. doi:10.1126/science.aac8469

51. Biagi E, Nylund L, Candela $M$ et al (2010) Through ageing, and beyond: gut microbiota and inflammatory status in seniors and centenarians. PLoS One 5(5):e10667. doi:10.1371/journal. pone. 0010667

52. Kong F, Hua Y, Zeng B, Ning R, Li Y, Zhao J (2016) Gut microbiota signatures of longevity. Curr Biol 26(18):R832-R833. doi:10.1016/j.cub.2016.08.015

53. Sansonetti PJ, Di Santo JP (2007) Debugging how bacteria manipulate the immune response. Immunity 26:149-161. doi:10.1016/j.immuni.2007.02.004

54. Schnorr SL, Candela M, Rampelli S et al (2014) Gut microbiome of the Hadza hunter-gatherers. Nat Commun 5:3654. doi: $10.1038 /$ ncomms 4654

55. Rampelli S, Schnorr SL, Consolandi C et al (2015) Metagenome sequencing of the Hadza hunter-gatherer gut microbiota. Curr Biol 25:1682-1693. doi:10.1016/j.cub.2015.04.055

56. De Filippo C, Cavalieri D, Di Paola M et al (2010) Impact of diet in shaping gut microbiota revealed by a comparative study in children from Europe and rural Africa. Proc Natl Acad Sci USA 107:14691-14696. doi:10.1073/pnas.1005963107

57. Yatsunenko T, Rey FE, Manary MJ et al (2012) Human gut microbiome viewed across age and geography. Nature 486:222227. doi:10.1038/nature 11053

58. Smits SA, Leach J, Sonnenburg ED et al (2017) Seasonal cycling in the gut microbiome of the Hadza hunter-gatherers of Tanzania. Science 357(6353):802-806. doi:10.1126/science.aan4834

59. Inzitari M, Doets E, Bartali B et al (2011) Nutrition in the agerelated disablement process. J Nutr Health Aging 15:599-604

60. Martha A, Christos S, Konstantinos M et al (2011) Age, weight and obesity. Maturitas 71:115-119. doi:10.1016/j. maturitas.2011.11.015

61. Wang F, Yu T, Huang G et al (2015) Gut microbiota community and its assembly associated with age and diet in Chinese centenarians. J Microbiol Biotechnol 25:1195-1204. doi:10.4014/ jmb.1410.10014

62. Roggenbuck D, Reinhold D, Baumgart DC et al (2016) Autoimmunity in Crohn's disease-a putative stratification factor of the clinical phenotype. Adv Clin Chem 77:77-101. doi:10.1016/ bs.acc.2016.06.002

63. Gu S, Chen Y, Zhang X et al (2016) Identification of key taxa that favor intestinal colonization of Clostridium difficile in an adult Chinese population. Microbes Infect 18:30-38. doi:10.1016/j. micinf.2015.09.008

64. Lange K, Buerger M, Stallmach A, Bruns T (2016) Effects of antibiotics on gut microbiota. Dig Dis 34:260-268. doi:10.1159/000443360

65. Rossen NG, Fuentes S, Boonstra K et al (2015) The mucosaassociated microbiota of PSC patients is characterized by low diversity and low abundance of uncultured Clostridiales II. J Crohns Colitis 9:342-348. doi:10.1093/ecco-jcc/jju023

66. Scher JU, Ubeda C, Artacho A et al (2015) Decreased bacterial diversity characterizes the altered gut microbiota in patients with psoriatic arthritis, resembling dysbiosis in inflammatory bowel disease. Arthritis Rheumatol (Hoboken NJ) 67:128-139. doi:10.1002/art.38892

67. Alam MZ, Alam Q, Kamal MA et al (2014) A possible link of gut microbiota alteration in type 2 diabetes and Alzheimer's disease pathogenicity: an update. CNS Neurol Disord Drug Targets $13: 383-390$ 
68. Tagliabue A, Elli M (2013) The role of gut microbiota in human obesity: recent findings and future perspectives. Nutr Metab Cardiovasc Dis 23:160-168. doi:10.1016/j.numecd.2012.09.002

69. Hullar MAJ, Lampe JW (2012) The gut microbiome and obesity. Nestle Nutr Inst Workshop Ser 73:67-79. doi: $10.1159 / 000341288$

70. Odamaki T, Kato K, Sugahara $\mathrm{H}$ et al (2016) Age-related changes in gut microbiota composition from newborn to centenarian: a cross-sectional study. BMC Microbiol 16:90. doi:10.1186/ s12866-016-0708-5

71. Montoliu I, Scherer M, Beguelin F et al (2014) Serum profiling of healthy aging identifies phospho- and sphingolipid species as markers of human longevity. Aging (Albany NY) 6:9-25. doi:10.18632/aging.100630

72. Cheng S, Larson MG, McCabe EL et al (2015) Distinct metabolomic signatures are associated with longevity in humans. Nat Commun 6:6791. doi:10.1038/ncomms7791

73. Cai D, Zhao S, Li D et al (2016) Nutrient intake is associated with longevity characterization by metabolites and element profiles of healthy centenarians. Nutrients. doi:10.3390/nu8090564

74. Ridlon JM, Bajaj JS (2015) The human gut sterolbiome: bile acid-microbiome endocrine aspects and therapeutics. Acta Pharm Sin B 5:99-105. doi:10.1016/j.apsb.2015.01.006

75. Ridlon JM, Harris SC, Bhowmik S et al (2016) Consequences of bile salt biotransformations by intestinal bacteria. Gut Microbes 7:22-39. doi:10.1080/19490976.2015.1127483

76. Petra AI, Panagiotidou S, Hatziagelaki E et al (2015) Gut-microbiota-brain axis and its effect on neuropsychiatric disorders with suspected immune dysregulation. Clin Ther 37:984-995. doi:10.1016/j.clinthera.2015.04.002

77. Schroeder BO, Bäckhed F (2016) Signals from the gut microbiota to distant organs in physiology and disease. Nat Med 22:10791089. doi: $10.1038 / \mathrm{nm} .4185$

78. Berer K, Mues M, Koutrolos M et al (2011) Commensal microbiota and myelin autoantigen cooperate to trigger autoimmune demyelination. Nature 479:538-541. doi:10.1038/nature10554

79. Hornig M (2013) The role of microbes and autoimmunity in the pathogenesis of neuropsychiatric illness. Curr Opin Rheumatol 25:488-795. doi:10.1097/BOR.0b013e32836208de

80. Skowrońska M, Albrecht J (2013) Oxidative and nitrosative stress in ammonia neurotoxicity. Neurochem Int 62:731-737. doi:10.1016/j.neuint.2012.10.013

81. Sheedy JR, Wettenhall REH, Scanlon D et al (2009) Increased D-lactic acid intestinal bacteria in patients with chronic fatigue syndrome. In Vivo 23:621-628

82. Qureshi MO, Khokhar N, Shafqat F (2014) Ammonia levels and the severity of hepatic encephalopathy. J Coll Physicians Surg Pak 24:160-163

83. Cryan JF, Dinan TG (2012) Mind-altering microorganisms: the impact of the gut microbiota on brain and behaviour. Nat Rev Neurosci 13:701-712. doi:10.1038/nrn3346

84. Galland L (2014) The gut microbiome and the brain. J Med Food 17:1261-1272. doi:10.1089/jmf.2014.7000

85. Freestone PPE, Haigh RD, Lyte M (2008) Catecholamine inotrope resuscitation of antibiotic-damaged Staphylococci and its blockade by specific receptor antagonists. J Infect Dis 197:10441052. doi:10.1086/529202

86. O'Mahony SM, Clarke G, Borre YE et al (2015) Serotonin, tryptophan metabolism and the brain-gut-microbiome axis. Behav Brain Res 277:32-48. doi:10.1016/j.bbr.2014.07.027

87. Kennedy PJ, Cryan JF, Dinan TG, Clarke G (2017) Kynurenine pathway metabolism and the microbiota-gut-brain axis. Neuropharmacology 112:399-412. doi:10.1016/j. neuropharm.2016.07.002
88. Stone TW, Darlington LG (2013) The kynurenine pathway as a therapeutic target in cognitive and neurodegenerative disorders. Br J Pharmacol 169:1211-1227. doi:10.1111/bph.12230

89. Keszthelyi D, Troost FJ, Masclee AA (2009) Understanding the role of tryptophan and serotonin metabolism in gastrointestinal function. Neurogastroenterol Motil 21:1239-1249. doi:10.1111/j.1365-2982.2009.01370.x

90. Kaszaki J, Erces D, Varga G et al (2012) Kynurenines and intestinal neurotransmission: the role of $N$-methyl-D-aspartate receptors. J Neural Transm 119:211-223. doi:10.1007/ s00702-011-0658-x

91. Heijtz RD, Wang S, Anuar F et al (2011) Normal gut microbiota modulates brain development and behavior. Proc Natl Acad Sci 108:3047-3052. doi:10.1073/pnas.1010529108

92. Clarke G, Grenham S, Scully P et al (2013) The microbiomegut-brain axis during early life regulates the hippocampal serotonergic system in a sex-dependent manner. Mol Psychiatry 18:666-673. doi:10.1038/mp.2012.77

93. Hsiao EY, McBride SW, Hsien S et al (2013) Microbiota modulate behavioral and physiological abnormalities associated with neurodevelopmental disorders. Cell 155:1451-1463. doi:10.1016/j.cell.2013.11.024

94. Yang Y, Sauve AA (2016) NAD + metabolism: bioenergetics, signaling and manipulation for therapy. Biochim Biophys Acta Proteins Proteom 1864:1787-1800. doi:10.1016/j. bbapap.2016.06.014

95. Verdin E (2015) NAD + in aging, metabolism, and neurodegeneration. Science (80-) 350:1208-1213. doi:10.1126/science. aac4854

96. Garrido A, Djouder N (2017) NAD + deficits in age-related diseases and cancer. Trends Cancer 3(8):593-610. doi:10.1016/j. trecan.2017.06.001

97. Quintana FJ, Sherr DH (2013) Aryl hydrocarbon receptor control of adaptive immunity. Pharmacol Rev 65:1148-1161. doi:10.1124/pr.113.007823

98. Pertovaara M, Raitala A, Lehtimäki T et al (2006) Indoleamine 2,3-dioxygenase activity in nonagenarians is markedly increased and predicts mortality. Mech Ageing Dev 127:497499. doi:10.1016/j.mad.2006.01.020

99. Zelante T, Iannitti RG, Fallarino F et al (2014) Tryptophan feeding of the IDO1-AhR axis in host-microbial symbiosis. Front Immunol 5:640. doi:10.3389/fimmu.2014.00640

100. Bessede A, Gargaro M, Pallotta MT et al (2015) Aryl hydrocarbon receptor control of a disease tolerance defence pathway. Nature 511:184-190. doi:10.1038/nature13323

101. Mezrich JD, Fechner JH, Zhang X et al (2010) An interaction between kynurenine and the aryl hydrocarbon receptor can generate regulatory T cells. J Immunol 185:3190-3198. doi:10.4049/ jimmunol.0903670

102. Rampelli S, Candela M, Turroni S et al (2013) Functional metagenomic profiling of intestinal microbiome in extreme ageing. Aging (Albany NY) 5:902-912. doi:10.18632/aging.100623

103. Badawy A (2017) Kynurenine pathway of tryptophan metabolism: regulatory and functional aspects. Int J Tryptophan Res 10:1178646917691938. doi:10.1177/1178646917691938

104. Brown-Borg HM, Buffenstein R (2016) Cutting back on the essentials: can manipulating intake of specific amino acids modulate health and lifespan? Ageing Res Rev 39:87-95. doi:10.1016/j.arr.2016.08.007

105. Welford RWD, Vercauteren M, Trébaul A et al (2016) Serotonin biosynthesis as a predictive marker of serotonin pharmacodynamics and disease-induced dysregulation. Sci Rep 6:30059. doi:10.1038/srep30059

106. Maddison DC, Giorgini F (2015) The kynurenine pathway and neurodegenerative disease. Semin Cell Dev Biol 40:134-141. doi:10.1016/j.semcdb.2015.03.002 
107. Reynolds GP, Pearson SJ (1989) Increased brain 3-hydroxykynurenine in Huntington's disease. Lancet (Lond Engl) 2:979-980

108. Sardar AM, Bell JE, Reynolds GP (1995) Increased concentrations of the neurotoxin 3-hydroxykynurenine in the frontal cortex of HIV-1-positive patients. J Neurochem 64:932-935

109. Ogawa T, Matson WR, Beal MF et al (1992) Kynurenine pathway abnormalities in Parkinson's disease. Neurology 42:1702-1706

110. Noristani HN, Verkhratsky A, Rodríguez JJ (2012) High tryptophan diet reduces CA1 intraneuronal $\beta$-amyloid in the triple transgenic mouse model of Alzheimer's disease. Aging Cell 11:810-822. doi:10.1111/j.1474-9726.2012.00845.x

111. Arosio B, Ostan R, Mari D et al (2017) Cognitive status in the oldest old and centenarians: a condition crucial for quality of life methodologically difficult to assess. Mech Ageing Dev 165(Pt B):185-194. doi:10.1016/j.mad.2017.02.010

112. Perls T (2004) Dementia-free centenarians. Exp Gerontol 39:1587-1593. doi:10.1016/j.exger.2004.08.015

113. Cattaneo A, Cattane N, Galluzzi S et al (2017) Association of brain amyloidosis with pro-inflammatory gut bacterial taxa and peripheral inflammation markers in cognitively impaired elderly. Neurobiol Aging 49:60-68. doi:10.1016/j. neurobiolaging.2016.08.019

114. Pistollato F, Sumalla Cano S, Elio I et al (2016) Role of gut microbiota and nutrients in amyloid formation and pathogenesis of Alzheimer disease. Nutr Rev 74:624-634. doi:10.1093/nutrit/ nuw023

115. Tremlett H, Bauer KC, Appel-Cresswell S et al (2017) The gut microbiome in human neurological disease: a review. Ann Neurol 81:369-382. doi:10.1002/ana.24901

116. Unger MM, Spiegel J, Dillmann K-U et al (2016) Short chain fatty acids and gut microbiota differ between patients with Parkinson's disease and age-matched controls. Parkinsonism Relat Disord 32:66-72. doi:10.1016/j.parkreldis.2016.08.019

117. Sampson TR, Debelius JW, Thron T et al (2016) Gut microbiota regulate motor deficits and neuroinflammation in a model of Parkinson's disease. Cell 167(1469-1480):e12. doi:10.1016/j. cell.2016.11.018

118. Felice VD, Quigley EM, Sullivan AM et al (2016) Microbiota-gut-brain signalling in Parkinson's disease: implications for non-motor symptoms. Parkinsonism Relat Disord 27:1-8. doi:10.1016/j.parkreldis.2016.03.012

119. Dinan TG, Cryan JF (2017) Gut instincts: microbiota as a key regulator of brain development, ageing and neurodegeneration. J Physiol 595:489-503. doi:10.1113/JP273106

120. Prenderville JA, Kennedy PJ, Dinan TG, Cryan JF (2015) Adding fuel to the fire: the impact of stress on the ageing brain. Trends Neurosci 38:13-25

121. Sudo N, Chida Y, Aiba Y et al (2004) Postnatal microbial colonization programs the hypothalamic-pituitary-adrenal system for stress response in mice. J Physiol 558:263-275. doi:10.1113/ jphysiol.2004.063388

122. Foster JA, McVey Neufeld K-A (2013) Gut-brain axis: how the microbiome influences anxiety and depression. Trends Neurosci 36:305-312. doi:10.1016/j.tins.2013.01.005

123. Caracciolo B, Xu W, Collins S, Fratiglioni L (2014) Cognitive decline, dietary factors and gut-brain interactions. Mech Ageing Dev 136:59-69. doi:10.1016/j.mad.2013.11.011

124. Bajaj JS, Ridlon JM, Hylemon PB et al (2012) Linkage of gut microbiome with cognition in hepatic encephalopathy. Am J Physiol Gastrointest Liver Physiol 302:G168-G175. doi:10.1152/ ajpgi.00190.2011

125. Neufeld KM, Kang N, Bienenstock J, Foster JA (2011) Reduced anxiety-like behavior and central neurochemical change in germ-free mice. Neurogastroenterol Motil 23:255-e119. doi:10.1111/j.1365-2982.2010.01620.x
126. Tillisch K, Labus J, Kilpatrick L et al (2013) Consumption of fermented milk product with probiotic modulates brain activity. Gastroenterology 144:1394-1401. doi:10.1053/j.gastro.2013.02.043 (1401e.1-4)

127. Liu X, Cao S, Zhang X (2015) Modulation of gut microbiotabrain axis by probiotics, prebiotics, and diet. J Agric Food Chem 63:7885-7895. doi:10.1021/acs.jafc.5b02404

128. Dinan TG, Stanton C, Cryan JF (2013) Psychobiotics: a novel class of psychotropic. Biol Psychiatry 74:720-726. doi:10.1016/j. biopsych.2013.05.001

129. De Filippis F, Pellegrini N, Vannini L et al (2016) High-level adherence to a Mediterranean diet beneficially impacts the gut microbiota and associated metabolome. Gut 65(11):1812-1821. doi:10.1136/gutjnl-2015-309957

130. MacFabe DF (2015) Enteric short-chain fatty acids: microbial messengers of metabolism, mitochondria, and mind: implications in autism spectrum disorders. Microb Ecol Health Dis 26:28177. doi:10.3402/mehd.v26.28177

131. David LA, Maurice CF, Carmody RN et al (2014) Diet rapidly and reproducibly alters the human gut microbiome. Nature 505:559-563. doi:10.1038/nature 12820

132. Martucci M, Ostan R, Biondi F, Bellavista E, Fabbri C, Bertarelli C, Salvioli S, Capri M, Franceschi C (2017) The mediterranean diet and inflammaging within the hormesis paradigm. Nutr Rev. doi:10.1093/nutrit/nux013

133. Derkinderen P, Shannon KM, Brundin P (2014) Gut feelings about smoking and coffee in Parkinson's disease. Mov Disord 29:976-979. doi:10.1002/mds.25882

134. Vaiserman AM, Koliada AK, Marotta F (2017) Gut microbiota: a player in aging and a target for anti-aging intervention. Ageing Res Rev 35:36-45. doi:10.1016/j.arr.2017.01.001

135. Zhang C, Li S, Yang L et al (2013) Structural modulation of gut microbiota in life-long calorie-restricted mice. Nat Commun 4:2163. doi: $10.1038 /$ ncomms 3163

136. Jonkers DMAE (2016) Microbial perturbations and modulation in conditions associated with malnutrition and malabsorption. Best Pract Res Clin Gastroenterol 30:161-172. doi:10.1016/j. bpg.2016.02.006

137. Rampelli S, Candela M, Severgnini M et al (2013) A probiotics-containing biscuit modulates the intestinal microbiota in the elderly. J Nutr Health Aging 17:166-172. doi:10.1007/ s12603-012-0372-x

138. Pérez Martínez G, Bäuerl C, Collado MC (2014) Understanding gut microbiota in elderly's health will enable intervention through probiotics. Benef Microbes 5:235-246. doi:10.3920/ BM2013.0079

139. Rondanelli M (2015) Review on microbiota and effectiveness of probiotics use in older. World J Clin Cases 3:156. doi:10.12998/wjcc.v3.i2.156

140. Saraswati S, Sitaraman R (2015) Aging and the human gut microbiota-from correlation to causality. Front Microbiol 5:764. doi:10.3389/fmicb.2014.00764

141. Thushara RM, Gangadaran S, Solati Z et al (2016) Cardiovascular benefits of probiotics: a review of experimental and clinical studies. Food Funct 7:632-642. doi:10.1039/C5FO01190F

142. Nakagawa H, Shiozaki T, Kobatake E et al (2016) Effects and mechanisms of prolongevity induced by Lactobacillus gasseri SBT2055 in Caenorhabditis elegans. Aging Cell 15:227-236. doi:10.1111/acel.12431

143. Kwon G, Lee J, Lim Y-H (2016) Dairy Propionibacterium extends the mean lifespan of Caenorhabditis elegans via activation of the innate immune system. Sci Rep 6:31713. doi:10.1038/srep31713

144. Grompone G, Martorell P, Llopis S et al (2012) Antiinflammatory Lactobacillus rhamnosus CNCM I-3690 strain protects against oxidative stress and increases lifespan in 
Caenorhabditis elegans. PLoS One 7:e52493. doi:10.1371/ journal.pone.0052493

145. Komura T, Ikeda T, Yasui $\mathrm{C}$ et al (2013) Mechanism underlying prolongevity induced by bifidobacteria in Caenorhabditis elegans. Biogerontology 14:73-87. doi:10.1007/s10522-012-9411-6

146. Park S-H, Kim K-A, Ahn Y-T et al (2015) Comparative analysis of gut microbiota in elderly people of urbanized towns and longevity villages. BMC Microbiol 15:49. doi:10.1186/ s12866-015-0386-8

147. Matsumoto M, Kurihara S, Kibe R et al (2011) Longevity in mice is promoted by probiotic-induced suppression of colonic senescence dependent on upregulation of gut bacterial polyamine production. PLoS One 6:e23652. doi:10.1371/journal.pone.0023652

148. Xu R, Shang N, Li P (2011) In vitro and in vivo antioxidant activity of exopolysaccharide fractions from Bifidobacterium animalis RH. Anaerobe 17:226-231. doi:10.1016/j.anaerobe.2011.07.010

149. Young VB, Hayden MK (2016) Environmental management in the gut: fecal transplantation to restore the intestinal ecosystem. Infect Dis (Lond Engl) 48:593-595. doi:10.1080/23744235.201 6.1177200

150. Rao K, Safdar N (2016) Fecal microbiota transplantation for the treatment of Clostridium difficile infection. J Hosp Med 11:5661. doi:10.1002/jhm.2449

151. Konturek PC, Haziri D, Brzozowski T et al (2015) Emerging role of fecal microbiota therapy in the treatment of gastrointestinal and extra-gastrointestinal diseases. J Physiol Pharmacol 66:483-491

152. Choi HH, Cho Y-S (2016) Fecal microbiota transplantation: current applications, effectiveness, and future perspectives. Clin Endosc 49:257-265. doi:10.5946/ce.2015.117

153. Vrieze A, Van Nood E, Holleman F et al (2012) Transfer of intestinal microbiota from lean donors increases insulin sensitivity in individuals with metabolic syndrome. Gastroenterology 143(913-916):e7. doi:10.1053/j.gastro.2012.06.031

154. Udayappan SD, Hartstra AV, Dallinga-Thie GM, Nieuwdorp M (2014) Intestinal microbiota and faecal transplantation as treatment modality for insulin resistance and type 2 diabetes mellitus. Clin Exp Immunol 177:24-29. doi:10.1111/cei.12293

155. Ananthaswamy A (2011) Faecal transplant eases symptoms of Parkinson's disease. New Sci 209:8-9

156. Turnbaugh PJ, Gordon JI (2009) The core gut microbiome, energy balance and obesity. J Physiol 587:4153-4158. doi:10.1113/jphysiol.2009.174136

157. Goodrich JK, Waters JL, Poole AC et al (2014) Human genetics shape the gut microbiome. PubMed commons. Cell 159:789799. doi:10.1016/j.cell.2014.09.053.Human

158. Goodrich JK, Davenport ER, Beaumont M et al (2016) Genetic determinants of the gut microbiome in UK twins. Cell Host Microbe 19:731-743. doi:10.1016/j.chom.2016.04.017

159. Garagnani P, Pirazzini C, Giuliani C et al (2014) The three genetics (nuclear DNA, mitochondrial DNA, and gut microbiome) of longevity in humans considered as metaorganisms. Biomed Res Int 2014:1-14. doi:10.1155/2014/560340
160. Moeller AH, Caro-Quintero A, Mjungu D et al (2016) Cospeciation of gut microbiota with hominids. Science 353:380-382. doi:10.1126/science.aaf3951

161. Groussin M, Mazel F, Sanders J et al (2017) Unraveling the processes shaping mammalian gut microbiomes over evolutionary time. Nat Commun 8:14319. doi:10.1038/ncomms14319

162. Bonder MJ, Kurilshikov A, Tigchelaar EF et al (2016) The effect of host genetics on the gut microbiome. Nat Genet 48:14071412. doi: $10.1038 / n g .3663$

163. Wang J, Thingholm LB, Skieceviciene J et al (2016) Genomewide association analysis identifies variation in vitamin $\mathrm{D}$ receptor and other host factors influencing the gut microbiota. Nat Genet 48:1396-1406. doi:10.1038/ng.3695

164. Kurilshikov A, Wijmenga C, Fu J, Zhernakova A (2017) Host genetics and gut microbiome: challenges and perspectives. Trends Immunol 38(9):633-647. doi:10.1016/j.it.2017.06.003

165. Liang L, Ai L, Qian J et al (2015) Long noncoding RNA expression profiles in gut tissues constitute molecular signatures that reflect the types of microbes. Sci Rep 5:11763. doi:10.1038/ srep11763

166. Liu S, da Cunha AP, Rezende RM et al (2016) The host shapes the gut microbiota via fecal microRNA. Cell Host Microbe 19:32-43. doi:10.1016/j.chom.2015.12.005

167. Capri M, Santoro A, Garagnani P et al (2014) Genes of human longevity: an endless quest? Curr Vasc Pharmacol 12(5):707-717

168. Xie H, Guo R, Zhong H et al (2016) Shotgun metagenomics of 250 adult twins reveals genetic and environmental impacts on the gut microbiome. Cell Syst. doi:10.1016/j.cels.2016.10.004

169. Flak MB, Neves JF, Blumberg RS (2013) Immunology. Welcome to the microgenderome. Science 339:1044-1045. doi:10.1126/ science. 1236226

170. Markle JGM, Frank DN, Mortin-Toth S et al (2013) Sex differences in the gut microbiome drive hormone-dependent regulation of autoimmunity. Science 339:1084-1088. doi:10.1126/ science. 1233521

171. Markle JG, Frank DN, Adeli K et al (2014) Microbiome manipulation modifies sex-specific risk for autoimmunity. Gut Microbes 5:485-493. doi:10.4161/gmic.29795

172. Org E, Mehrabian M, Parks BW et al (2016) Sex differences and hormonal effects on gut microbiota composition in mice. Gut Microbes 7:313-322. doi:10.1080/19490976.2016.1203502

173. Wallis A, Butt H, Ball M et al (2016) Support for the microgenderome invites enquiry into sex differences. Gut Microbes 976:1-7. doi:10.1080/19490976.2016.1256524

174. Meighani A, Hart BR, Mittal C et al (2016) Predictors of fecal transplant failure. Eur J Gastroenterol Hepatol 28:826-830. doi:10.1097/MEG.0000000000000614

175. Dominianni C, Sinha R, Goedert JJ et al (2015) Sex, body mass index, and dietary fiber intake influence the human gut microbiome. PLoS One 10:1-14. doi:10.1371/journal.pone.0124599

176. Bolnick DI, Snowberg LK, Hirsch PE et al (2014) Individual diet has sex-dependent effects on vertebrate gut microbiota. Nat Commun 5:4500. doi:10.1038/ncomms5500 